\title{
Generalized Shields criterion for weakly cohesive granular materials
}

\author{
Florian Brunier-Coulin, ${ }^{1}$ Pablo Cuéllar, ${ }^{2}$ and Pierre Philippe $\odot^{3}$ \\ ${ }^{1}$ Aix Marseille Univ, Centrale Marseille, IRPHE UMR 7342, 13384 Marseille, France \\ ${ }^{2}$ BAM, Division 7.2 Buildings and Structures, Unter den Eichen 87, 12205 Berlin, Germany \\ ${ }^{3}$ Irstea, Aix Marseille Univ, RECOVER, 3275 Route Cézanne, 13182 Aix-en-Provence, France
}

(Received 18 June 2019; accepted 26 February 2020; published 30 March 2020)

\begin{abstract}
The erosion of natural sediments by a superficial fluid flow is a generic situation in many usual geological or industrial contexts. However, there is still a lack of fundamental knowledge about erosional processes, especially concerning the role of internal cohesion and adhesive stresses on issues such as the critical flow conditions for the erosion onset or the kinetics of soil mass loss. This contribution investigates the influence of cohesion on the surface erosion by an impinging jet flow based on laboratory tests with artificially bonded granular materials. The model samples are made of spherical glass beads bonded either by solid bridges made of resin or by liquid bridges made of a highly viscous oil. To quantify the intergranular cohesion, the capillary forces of the liquid bridges are here estimated by measuring their main geometrical parameters with image-processing techniques and using well-known analytical expressions. For the solid bonds, the adhesive strength of the materials is estimated by direct measurement of the yield tensile forces and stresses at the particle and sample scales, respectively, with specific traction tests developed for this purpose. The proper erosion tests are then carried out in an optically adapted device that permits a direct visualization of the scouring process at the jet apex by means of the refractive index matching technique. On this basis, the article examines qualitatively the kinetics of the scour crater excavation for both scenarios, namely, for an intergranular cohesion induced by either liquid or solid bonds. From a quantitative perspective, the critical condition for the erosion onset is discussed specifically for the case of the solid bond cohesion. In this respect, we propose here a generalized form of the Shields criterion based on a common definition of a cohesion number from yield tensile values, derived at both micro- and macroscales. The article finally shows that the proposed form manages to reconcile the experimental data for cohesive and cohesionless materials, the latter in the form of the so-called Shields curve along with some previous results of the authors which have been appropriately revisited.
\end{abstract}

DOI: 10.1103/PhysRevFluids.5.034308

\section{INTRODUCTION}

In its broadest sense, erosion refers to the gradual removal of solid matter from the surface of a material under the action of an eroding agent such a fluid flow. This definition covers a very wide range of situations, for instance, in terms of the eroding flow (precipitation run-off, wind gusts, waterfall, flood tide, fluvial stream, etc.), eroding fluid (water, air, mud, etc.), and eroded material (rocks, snow, marine muddy sediments, sand, clayey soils, etc.). The associated temporal and spatial scales can vary widely, from geological ages to sudden collapses (e.g., of hydraulic infrastructure) and large canyons to local scouring. In nature, erosion also causes sediment transport and its subsequent deposition, which are deeply related to a myriad of issues, potential risks, and challenges: e.g., the gradual decline of the coastline and loss of sandy beaches by marine erosion or due to increased flooding as a result of the ongoing climatic change (rise of sea level and the 
foreseeable intensification of storms) [1], the dispersion of pollutants such as radionuclides after the Fukushima nuclear power plant accident in 2011 [2], the prediction of estuary and river bed evolution [3,4], the erosion on hill slopes in active rills [5] or the occurrence of gully erosion [6]. In addition, the anthropogenic soil erosion produced by agricultural activities has also been reported to have an impact on the global carbon cycle [7]. In the the context of civil infrastructures, many constructions are also threatened by erosional degradation such as the scouring of soil surrounding bridge foundations (piers and abutments) [8], the excavated holes caused by the impinging water flow from dam spillways [9], or the erosion-related failure of hydraulic earthworks such as dams, levees, and dikes [10-13].

This all highlights the importance of an accurate quantification of the resistance of soils to surface erosion. Despite the active research on this subject, our knowledge about soil erosion remains still partial and mainly empirical. The reason for this lies in the complexity of the phenomenon, with respect both to the hydrodynamical conditions, usually involving fluid flows in turbulent or transitional regimes and large stress fluctuations, and to the soil's nature, often made out of particles within a wide range of sizes, shapes, and material properties, including cohesive behavior in varying degrees.

For strictly noncohesive materials, such as typical sands or gravels, the resistance against erosion is relatively well understood since the pioneering work of A. F. Shields $[3,14,15]$. The onset of erosion of an individual particle can be deduced from a critical value of the dimensionless Shields number $\mathrm{Sh}_{0}$, defined as the ratio between the fluid shear stress $\tau$, estimated from the local shear velocity, and the buoyant weight of the particle. This critical value varies by less than one order of magnitude as a function of the shear Reynolds number $\operatorname{Re}_{\tau}$, constructed with the local shear velocity and the particle diameter [14]. A possible explanation of the observed experimental dispersion may be found in certain aging processes involving subcritical shear flows, such as consolidation and armoring [16,17]. A trend curve was proposed on the so-called Shields diagram [14], and many explicit empirical equations exist for the critical Shields number $[15,18,19]$. In this respect, some authors argue that lower threshold values can be systematically obtained if the criterion for erosion is determined by a torque condition instead of the usual force balance [20,21]. As a further alternative, Sutherland and Dalziel found that the Rouse number (i.e., the particle settling velocity divided by the centerline jet velocity) could be better suited than the Shields number to account for the crater formation onset in their study of sediments impinged by a turbulent jet [22].

However, the situation is more complex for the case of cohesive materials, such as cemented calcareous sands, clayey soils, or benthic sediments. In this case, there is no longer a clear picture for the particles' mobilization by a fluid flow, and the original Shields approach becomes invalid. In this respect, the critical shear stress $\tau_{c}$ is here dependent not only on friction and buoyant weight but also on the attractive/repulsive interactions between the particles and on the resulting internal adhesive forces [23-26]. For common natural soils, this internal cohesion is often a relevant factor (if not the dominant one) for the magnitude of $\tau_{c}$.

Concerning the kinetics of the erosive process at a macroscopic scale, at least one further parameter needs to be quantified in this case, since the erosion of cohesive media necessarily involves the breakage of a number of interparticle bonds for the removal of a variable-sized aggregate by the fluid flow. Such complexity calls for a better understanding of the elementary mechanisms of cohesive soils erosion [4]. However, given the scarcity of related physical insights, the empirical approaches have traditionally been favored in the scientific community, notably through the elaboration of erosion laws [27]. The most popular one, originally proposed by Parteniades [28] and later by Ariathurai and Arulanandan [29], assumes that, locally and for an homogeneous sample, the rate of erosion (expressed either as a mass loss per surface and per second or as a thickness loss per second) is proportional to the excess shear stress, i.e., $\tau-\tau_{c}$ with $\tau$ the fluid shear stress and $\tau_{c}$ the previously mentioned resistant stress. The proportionality coefficient of this relationship, often denoted $k$ as the erosion rate parameter, consequently rules the kinetics of erosion. In this respect it is also worth noting that the assumption of linear proportionality has been replaced by a power-law relationship by some authors, e.g., Ref. [30], which permits, for instance, the unification of several expressions that have been proposed for sand-mud mixtures [25] or for 
stratified beds of fine sediments [31]. Also, other alternative forms, not exclusively based on the fluid shear stress, have also been proposed by some authors $[5,32,33]$.

Despite its very simple form, the linear formulation with the excess shear stress often shows acceptable agreement with the experimental data (see, for instance, Ref. [34] or [35]). It also permits a classification of soils by systematic evaluation of the two erosion (or erodibility) parameters, $\tau_{c}$ and $k$, by means of specific erosion tests such as the Erosion Function Apparatus (EFA) [36], the Hole Erosion Test (HET) [37], or the Jet Erosion Test (JET) [38]. The last is probably the device most widely used due to its suitability for experiments both in the laboratory and in the field, and with all types of soil irrespective of their degree of cohesion.

In the present study, we focus specifically on the onset of erosion by an adjacent fluid flow for several cohesive granular soils and propose a way to extend the applicability of the Shields criterion by addition of a characteristic cohesive stress (based here on tensile strengths) to the soil's resistant stress. This approach goes in line with previous proposals for the generalization of the Shields number; see, e.g., Refs. [25,26,39-42]. As already suggested in these references, it appears relevant to compare here the cohesion force, i.e., the mean bond strength, to the particle's buoyant weight. We will consistently introduce in what follows a dimensionless cohesion number $\mathrm{Co}$, also previously referred to as cohesive granular Bond number $\mathrm{Bo}_{g}$ [43-45], while noting that $\mathrm{Bo}_{g}$ is in fact the inverse of the usual Bond number definition in fluid mechanics when the cohesion is substituted by the surface tension stress.

The discussion is here based on an experimental study of artificial granular materials obtained by mixing glass beads with different cohesive matrices, either solid or viscous, leading to two distinct types of cohesion. A liquid capillary bond between two particles can actually be highly strained in tension before rupture, while being barely affected by tangential displacements at the contact point. Moreover, the liquid bonds can also generally be rebuilt hysteretically by coalescence after prior breakage. In contrast, the solid cohesive bonds in the present range of millimetric particle sizes usually break irreversibly with much less deformation and often at a much higher tensile stress level, while also being capable of sustaining significant shear stresses. Regarding the cemented materials made out of grains bonded by such solid bridges, it is possible to identify different kinds of bond breakage. In this sense, a crack at a given bond can propagate either inside the solid bridge or along its interface with one of the connected particles. These two cases are often termed cohesive and adhesive failures, respectively [46]. A third type of bond failure, where the crack penetrates partially or completely inside the particles [47], is also possible but irrelevant here since the damage limit of our glass beads is substantially higher than that of the solid matrix. Therefore, neither surface abrasion nor particle breakage [47] is expected in the present investigation with the type of cohesive bridges used in our experiments and described hereafter. The resulting cohesive granular materials were then subjected to mechanical tests at both local and sample scales for the assessment of their cohesive strength. Finally, the erodibility of the samples was evaluated quantitatively by means of JET tests (i.e., an immersed impinging jet as the fluid flow configuration) and ultimately compared to a previous analysis by the authors in the limit of the cohesionless case [48]. Special attention was devoted to the choices of materials and methods, which in this case permits the use of a nonintrusive optical probe in order to investigate not only the erosion threshold but also the subsequent crater scouring and its kinetics. To account for the different degrees of internal cohesion in relation to the amount of bond matrix added to the granular material, we propose here to characterize the cohesion quantitatively based on tensile strength measurements.

The remainder of this paper is organized as follows: Sec. II revisits previous results by the authors [48] in the light of recent numerical results [49] for an accurate estimate of fluid shear stress for an impinging jet. Next, Sec. III is devoted to the description of materials and methods, dealing consecutively with (i) the employed artificial systems, made out of glass beads mixed with either viscous or solid cohesive bonds; (ii) the specific tensile strength measurements at both particle and sample scales for the assessment of the cohesion strength; and finally (iii) an optically adapted version of the common impinging jet erosion test (RIM-JET). On the basis of the fluid shear stress expression given in Sec. II, the results obtained from the RIM-JET tests are then discussed in 
Sec. IV, successively for cohesion/adhesion induced by liquid and solid bonds, in terms both of the critical condition for erosion onset and of the subsequent scouring kinetics. Finally, the onset of erosion is further analyzed in Sec. V with the introduction of a cohesion number Co. In this respect, we propose a generalized form of the Shields number Sh that relates in a simple manner the classical cohesionless Shields number $\mathrm{Sh}_{0}$ to $\mathrm{Co}$. As a closure, the main conclusions of this study and some open perspectives are briefly summarized in Sec. VI.

\section{REVISITING THE DETERMINATION OF EROSION ONSET}

\section{A. Relevance of the free jet model}

Although submitting a soil specimen to an impinging fluid jet has proved to be a simple and versatile way to initiate soil erosion, both in the laboratory or in situ [38], the related hydrodynamic flow is actually quite complex. It combines the inherent complexity of the jet flow with that of the impingement on a surface, which is initially flat but then gets progressively scoured by erosion leading to a changing geometry. In this respect, one of the main challenges is to relate the maximal shear-stress exerted by the impinging flow at the soil's surface to the few inlet conditions and geometrical parameters. In almost all previous studies on soil erosion by an impinging jet, this issue is circumvented by using empirical expressions derived from experiments of solid wall impingements [38,50,51]. In some instances this approach is improved to account for the flow confinement [52] or coupled with even stronger assumptions such as directly neglecting the presence of the soil surface to permit the use of well-known self-similar free jet models $[48,53,54]$. In the related literature, several numerical analyses of the impinging jet flow have been proposed for both laminar and turbulent flow regimes, while the actual coupling to soil erosion may also be included either explicitly or implicitly with different strategies. In this respect, the jet erosion may be modeled in a monophasic approach with an Eulerian flow description and a Lagrangian water/soil boundary condition that is continuously updated in an explicit manner based on a prescribed erosion law [55]. Alternatively, the biphasic analytical approaches, for instance, with an Eulerian flow description and Lagrangian model of the soil as discrete interacting particles [56-58], are often more demanding computationally but can reproduce soil erosion without requiring any phenomenological assumption about the erosion itself.

A recent numerical study by some of the authors using the lattice Boltzman method (LBM) has highlighted the relevance of the free jet models to quantify the maximal shear-stress $\tau_{m}$ at the soil surface for 2D scenarios of laminar jets impinging on either solid or granular surfaces [49]. There the derived expression for the maximal shear stress includes a Blasius-like friction term, i.e., a friction coefficient inversely proportional to the square root of the flow Reynolds number as observed for a laminar flow over a flat plate [54]. In particular, $\tau_{m}$ is obtained symmetrically at both sides and at a certain distance from the jet axis, while its magnitude can be simply approximated by [49]

$$
\tau_{m}=\frac{1}{2} C_{f} \rho_{l} v_{m}^{2},
$$

where the maximal horizontal velocity $v_{m}$ along the soil surface was found to be directly proportional to the maximal vertical velocity $\widetilde{u}_{2 D}\left(h_{0}\right)$ predicted from a classical $2 \mathrm{D}$ laminar free jet model [54] at a distance $h_{0}$ from the outlet. In practice, the distance to be considered here is not exactly $h_{0}$ but $h_{0}+\lambda$ where $\lambda$ stands for the distance from the nozzle to the virtual origin of the jet, i.e., the source point from which the jet appears to emerge when observed sufficiently far from the outlet [59]. As mentioned above, the friction factor $C_{f}$ obtained numerically is reminiscent of a Blasius friction law and reads

$$
C_{f}=\frac{1.53}{\sqrt{\operatorname{Re}_{J}}}
$$

with $\operatorname{Re}_{J}$ denoting the jet Reynolds number.

In the light of these recent findings, the previous experimental results by Badr et al. [53] on the erosion onset of a granular bed by a plane-impinging jet have been revisited, leading to more 
realistic values of the critical Shields number [49]. The following section follows a similar approach in the present 3D situation and compares it with the recently published results carried out with cohesionless materials using the same experimental device [48].

\section{B. Reanalysis of the cohesionless case studied in Brunier-Coulin et al. [48]}

Considering the strong similarity of laminar flow conditions in the 2D numerical calculations of Benseghier et al. [49] and in the present 3D experimental configuration, it appears reasonable to assume here as well a Blasius-type friction law for a laminar round jet impingement, $C_{f} \propto$ $\operatorname{Re}_{J}^{-1 / 2}$, and then propose the following expression for the maximal bed shear stress $\tau_{m}$, by direct generalization from $2 \mathrm{D}$ to $3 \mathrm{D}$ :

$$
\tau_{m}=a \operatorname{Re}_{J}^{-1 / 2} \rho_{l}\left[\widetilde{u}_{3 D}\left(h_{0}\right)\right]^{2},
$$

where $\tilde{u}_{3 D}\left(h_{0}\right)$ is the maximal vertical velocity predicted from a classical laminar free round jet model [54] at a distance $h_{0}$ and accounting for the previously mentioned virtual origin $\lambda$.

In contrast to Ref. [48] or [53], a complete definition of the cohesionless Shields number can be used now,

$$
\mathrm{Sh}_{0}=\frac{\tau_{m}}{\left(\rho_{g}-\rho_{l}\right) g d}
$$

where $\rho_{g}$ stands for the grain density, $\rho_{l}$ for liquid density, $d$ for (mean) grain diameter, and $g$ for gravity.

As presented in the Appendix, the coefficient $a$ can be found by minimizing the mean-squared error when the experimental values of the critical Shields numbers $\mathrm{Sh}_{0}^{*}$ recalculated from Brunier's data set [48] are compared to their corresponding values given by Guo's explicit formulation of the Shields curve [18]. This fitting process yields finally $a=0.30 \pm 0.07$ with a substantial relative uncertainty of $23 \%$. Ultimately, the complete expression of the maximal shear stress at the soil surface in our RIM-JET device (see Sec. III C below) reads

$$
\tau_{m}=0.00469 \frac{\rho_{l} v_{l}^{2} \operatorname{Re}_{J}^{7 / 2}}{\left(h_{0}+0.048 \operatorname{Re}_{J} D_{J}\right)^{2}} .
$$

This final expression will be used hereafter in Secs. IV and V to derive the critical values for the fluid shear stress and the corresponding Shields number as a function of the cohesion strength and Reynolds number.

\section{EXPERIMENTAL METHODOLOGY}

\section{A. Preparation of optically adapted artificial materials with cohesion}

A direct visualization of the grains' microstructure within a fully saturated particulate medium can be obtained, for instance, by combination of two optical techniques: the Refractive Index Matching (RIM) and the Planar Laser Induced Fluorescence (PLIF). The RIM consists in adjusting the refractive index of the interstitial liquid with the one of the solid particles. Thus, after full immersion, the interfaces between the liquid and solid phases become optically undistinguishable making the whole medium translucent. After prior addition of a fluorescent dye to the liquid phase, the PLIF allows one to visualize a chosen 2D region within the RIM system by illumination with a planar laser sheet. Due to the characteristic wavelength shift of fluorescent emissions, the use of an optical filter permits the removal of most of the primary light from the laser beam and consequently obtain typical high-contrast images with the liquid phase in bright and the solid phase in dark. The combined use of the RIM and PLIF techniques has already been used for nonintrusive probes, specifically within fully saturated granular materials [60] but also in more general multiphase systems [61]. 

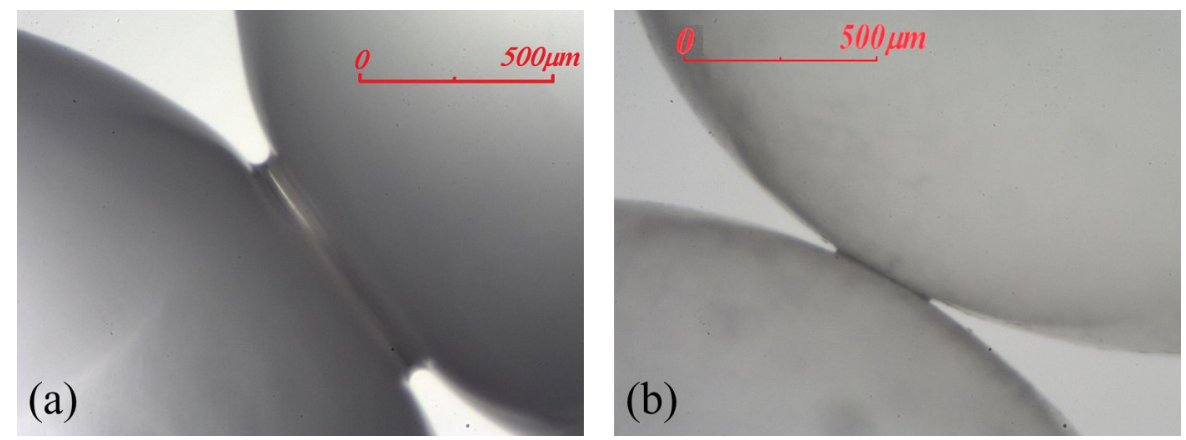

FIG. 1. (a) Capillary bridge of Ucon oil between two borosilicate glass beads of diameter $d=3 \mathrm{~mm}$ with a Ucon oil mass content $\zeta_{u}=0.5 \%$. (b) Solid bridge of dried polyurethane resin between two borosilicate glass beads of diameter $d=3 \mathrm{~mm}$ with an initial resin mass content $\zeta_{r}=0.2 \%$.

To implement this technique in a cohesive granular medium, we chose to create the adhesive bonds between particles with a transparent viscous matrix featuring a similar refractive index to that of the two other phases. In practice, since this matrix is added in a small quantity, a fine adjustment of its refractive index is not necessary and the slight mismatch does not disturb substantially the overall optical matching. In this respect, the solid particles and interstitial liquid used here are actually the same as used in previous studies by the authors $[48,62,63]$. The particles are spherical borosilicate glass beads (manufactured by SiLi Beads) of diameter $3.0 \pm 0.02 \mathrm{~mm}$ and density $\rho_{b}=$ $2230 \mathrm{~kg} \mathrm{~m}^{-3}$. The RIM liquid is an oil mixture made up of $90 \%$ in mass fraction of light mineral oil (provided by Sigma-Aldrich) and 10\% of immersion oil type A (manufactured by Cargille). Red Nile dye (supplied by Fulka), added in a very small quantity, was chosen as a convenient fluorescent dye, miscible in our oil mixture and adapted to a $532 \mathrm{~nm}$ laser wavelength. The following properties of the oil mixture were measured at $23^{\circ} \mathrm{C}$ : its density was estimated to $\rho_{l}=846 \pm 5 \mathrm{~kg} \mathrm{~m}^{-3}$ while its viscosity was measured to $\mu_{l}=28 \pm 2 \mathrm{cP}$ with a Falling Ball Viscometer (manufactured by Gilmont), and the matched refractive index, evaluated with a refractometer (model Master-RI provided by Atago), is $n_{l}=1.472 \pm 0.001$. Note that the optical matching, which was simply adjusted visually, is fully consistent with the typical range 1.470-1.474 given for the refractive index of borosilicate beads in the literature [61].

Two other sets of particles were also used for this study: silica glass beads with diameters ranging between 2.85 and $3.30 \mathrm{~mm}$ and between 0.75 and $1 \mathrm{~mm}$. The density of the silica glass was measured to $\rho_{s}=2495 \mathrm{~kg} \mathrm{~m}^{-3}$. These beads have a higher value of refractive index than the borosilicate one and thus a worse optical matching with the oil mixture. Consequently, they do not allow anymore for RIM-PLIF visualization, and their use was restricted to the evaluation of the erosion onset, by observation from the top surface, according to the first protocol described later in Sec. III C.

A global cohesion for such granular media is obtained by adding in small quantity a transparent matrix to glue the particles together before the immersion by the RIM liquid. As already mentioned, two different cohesive matrices were used in order to create either liquid or solid bonds, as illustrated in Fig. 1, which represent two alternative types of cohesive behavior.

The liquid bonds are made of a Newtonian liquid of very high viscosity, namely, Ucon oil (UCON $^{\mathrm{TM}}$ Lubricant $75-\mathrm{H}-90,000$, provided by Dow Chemical Company). According to the commercial datasheet, its kinematic viscosity is approximatively $17000 \mathrm{cSt}$ (at $40^{\circ} \mathrm{C}$ ), its density $1090 \mathrm{~kg} \mathrm{~m}^{-3}$ (at $20^{\circ} \mathrm{C}$ ), and its surface tension in air $\gamma_{u a} \approx 51 \mathrm{mN} \mathrm{m}^{-1}$ (at $25^{\circ} \mathrm{C}$ ). A measure of its refractive index with our refractometer gave a value $1.464 \pm 0.001$ (at $23^{\circ} \mathrm{C}$ ) that is indeed rather close to that of the borosilicate. Moreover, Ucon oil is conveniently not miscible with the RIM oil mixture. A small quantity of this viscous matrix can be added and almost uniformly distributed within a beads sample (in air) without notably perturbing the global transparency after full immersion into the RIM liquid. To this end, samples with a given mass of beads were 

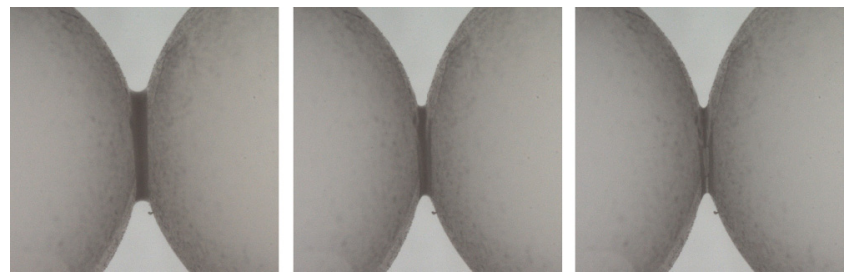

FIG. 2. Drying process at ambient conditions from liquid to solid bond made of a commercial resin (Syntilor), with initial resin mass content $\zeta_{r}=0.1 \%$, between two beads of diameter $7 \mathrm{~mm}$ at successive times $t=0,4$, and 8 min shown from left to right.

prepared by stirring manually a small amount of Ucon oil with a long metal rode, forming almost homogeneously capillary bridges at each contact between two beads as shown in the left picture of Fig. 1, captured with an optical zoom (Navitar Zoom 6000). The initial mass fraction of Ucon oil $\zeta_{u}$ varied between $0.2 \%$ and $1 \%$, giving rise to different sizes of bonds and consequently to samples with potentially different apparent cohesion, as will be discussed later in Sec. V.

The solid bonds are made of a transparent polyurethane resin (supplied by Syntilor). In practice, the resin was initially liquid and mixed in small quantity with a beads sample to create interparticle capillary bridges as for Ucon oil and using the same protocol. Then the sample was dried during several days in a controlled warming chamber at $60^{\circ} \mathrm{C}$. An example of such a solid bond between two particles is presented in the right panel of Fig. 1. To vary the amount of resin while maintaining a reproducible way to prepare the system, we chose to keep constant the initial volume of liquid added to the beads while using a mixture of resin and distilled water in a proportion up to $100 \%$ in resin. For most of our samples made of a beads mass $M_{b}=3 \mathrm{~kg}$, the added liquid volume was fixed to $V_{l}=V_{w}+V_{r}=6 \mathrm{~cm}^{3}$. The volume of resin was chosen in the range $0.85 \mathrm{~cm}^{3}<V_{r}<6.0 \mathrm{~cm}^{3}$, corresponding to a mass content in resin $0.05<\zeta_{r}<0.2 \%$ with respect to the initial liquid resin. Figure 2 shows that there was a significant volume reduction during drying, mostly due to water evaporation: both the distilled water volume $V_{w}$ added to the liquid resin volume $V_{r}$ to reach the target volume $V_{l}$ but also the water initially present in the liquid resin supplied by the manufacturer and whose mass fraction was measured equal to around $65 \%$ after complete drying and subsequent weighting. The volume fraction of the resin was measured at $23^{\circ} \mathrm{C}$, either in liquid phase or in solid phase, after complete drying, and found approximately equal to $\rho_{r}^{l}=1,004 \mathrm{~kg} \mathrm{~m}^{-3}$ and $\rho_{r}^{s}=$ $1115 \mathrm{~kg} \mathrm{~m}^{-3}$, respectively. In the end, the overall volume reduction from initial liquid bridges to final solid resin bonds was very large, ranging from $65 \%$ up to $95 \%$. Paraffin wax was initially envisaged as an alternative bonding substance which presents the advantages of a much smaller volume change and a quicker hardening. However, the solid paraffin bridges are naturally removed from the glass particles in a short time after immersion in the RIM-liquid due to an unfavorable surface energy balance.

These artificial materials were then subjected to mechanical and JET tests with different experimental devices developed ad hoc. As detailed below, the tensile strength of the cohesive samples with solid bonds was tested in both micro- and macromechanical tensile setups, while the jet erodibility of both the liquid and solid bonded materials was investigated by means of impinging jets in RIM conditions. An overview of all the tests conducted in the experimental campaign for the present study can be found in the Supplemental Material to this paper [64].

\section{B. Quantification of cohesive strength}

\section{Liquids bonds}

The internal cohesive strength generated by the liquid bonds of Ucon oil is due to capillary forces, restricted here to the pendular regime where liquid bridges exist only in the shape of individual pendular necks between two neighboring particles [65]. In general, and denoting $\gamma$ the surface 


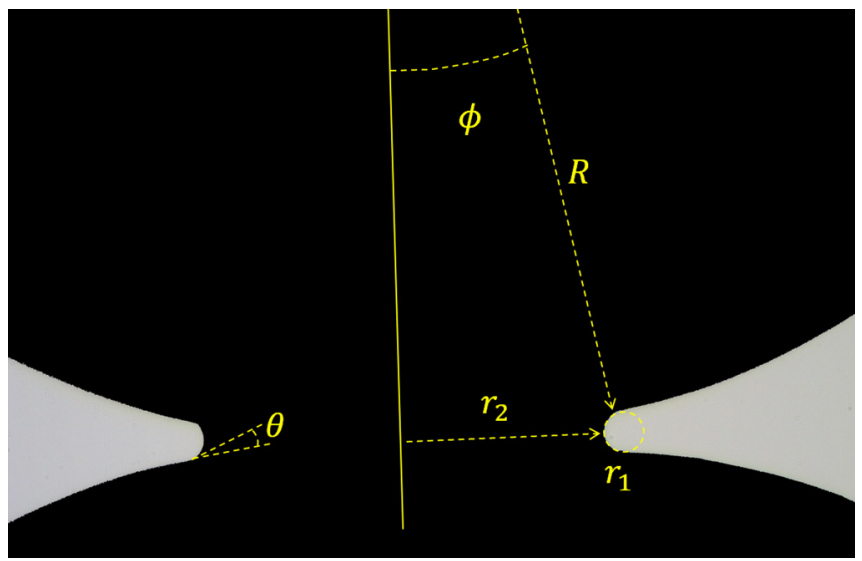

FIG. 3. Typical capillary bridge profile deduced from a picture (similar to the left one in Fig. 1) after some image processing using Image J. This figure defines the radius of curvature of the meniscus $r_{1}$, the radius of the capillary bridge $r_{2}$, the half-filling angle $\phi$, and the contact angle $\theta$.

tension between the liquid in a bridge and the surrounding fluid, the attractive force $F_{\text {cap }}$ between two particles connected by a capillary bridge can be expressed analytically. Assuming that influence of gravity on the capillary bridge can be neglected [66], the exact value of $F_{\text {cap }}$ depends on the shape and volume of the bridge, the radii of the two particles, and the Young-Laplace pressure, given by the surface tension $\gamma$ and the local curvature [66-70]. The capillary force of a liquid bridge can also be approximated by the so-called "gorge method" [67,69], which gives

$$
F_{\text {cap }}=\pi \gamma r_{2}\left(1+\frac{r_{2}}{r_{1}}\right) \text {. }
$$

In this equation, $r_{1}$ and $r_{2}$ stand for the radius of curvature of the meniscus and the radius of the capillary bridge, respectively, and are given by the following expressions for the case of two equal spheres of radius $R$ :

$$
r_{1}=\frac{R(1-\cos \phi)}{\cos (\phi+\theta)}
$$

and

$$
r_{2}=R \sin \phi-[1-\sin (\phi+\theta)] r_{1},
$$

where $\phi$ is the half-filling angle and $\theta$ the contact angle.

Figure 3, which was derived from a close-up picture after some postprocessing with the software Image $\mathrm{J}$, illustrates the geometrical definitions for $r_{1}, r_{2}, \phi$, and $\theta$. From this kind of processed image, it is possible to estimate the capillary force $F_{\text {cap }}$ using Eqs. (6)-(8), owing that $\phi, \theta, r_{1}$, and $r_{2}$ can be estimated with enough precision. To this end, these parameters were evaluated in air for each value of Ucon oil mass content, namely, $\zeta_{u}=0.2 \%, 0.3 \%, 0.4 \%, 0.5 \%$, and $1 \%$, on many different liquid bridges taken randomly in a sample prepared with the protocol previously described in Sec. III A.

Among these geometrical quantities, the contact angle $\theta$ and the radius of curvature $r_{1}$ of the meniscus are the most difficult to evaluate with accuracy (see Fig. 3). Preliminary measurements of $\theta$ showed a mean value around $16^{\circ}$ with an almost random distribution from 0 to about $30^{\circ}$, irrespective of $\zeta_{u}$. Since $\theta$ is in theory a constant value given by the Young-Dupré equation, we chose to adopt a fixed value of $\theta=16^{\circ}$. Note that imposing alternatively a perfect wetting condition (i.e., $\theta=0^{\circ}$ ) has only a limited impact on the value of the capillary force, with a slight increase of approximately $4 \%$ as compared to the chosen value of $16^{\circ}$. From typical pictures as illustrated in 
TABLE I. Geometrical parameters, obtained by statistically averaging over 10 capillary bridges, as a function of the Ucon oil mass content $\zeta_{u}$ and for $3 \mathrm{~mm}$ borosilicate glass beads. The half-filling angle $\phi$ (second column) and the radius of the capillary bridge $r_{2}$ (third column) are measured. The radius of curvature of the meniscus $r_{1}$ (fourth column) is deduced from Eq. (7) using $\theta=16^{\circ}$ as contact angle. The radius of the capillary bridge $r_{2}$ (fifth column) is alternatively obtained analytically from Eq. (8) with the same value for $\theta$.

\begin{tabular}{lcccc}
\hline \hline$\zeta_{u}(\%)$ & $\phi\left(^{\circ}\right)$ & $r_{2}(\mu \mathrm{m})$ & $r_{1}(\mu \mathrm{m})$ & $r_{2}(\mu \mathrm{m})$ \\
\hline 0.2 & $6.1 \pm 1.7$ & $162 \pm 46$ & $9.8 \pm 7.0$ & $153 \pm 41$ \\
0.3 & $9.0 \pm 4.0$ & $234 \pm 97$ & $24.9 \pm 25.6$ & $222 \pm 90$ \\
0.4 & $8.0 \pm 1.0$ & $208 \pm 29$ & $16.3 \pm 4.0$ & $200 \pm 23$ \\
0.5 & $10.2 \pm 1.9$ & $251 \pm 42$ & $27.2 \pm 10.3$ & $250 \pm 44$ \\
1.0 & $13.6 \pm 1.7$ & $342 \pm 43$ & $48.9 \pm 13.6$ & $327 \pm 38$ \\
\hline \hline
\end{tabular}

Fig. 3, it was possible to measure the half-filling angle $\phi$ and the radius of the capillary bridge $r_{2}$ with reasonable accuracy. The corresponding values are reported in Table I. For instance, the mean value of $\phi$ increases with the Ucon oil mass content $\zeta_{u}$ from $6^{\circ}$ to $13^{\circ}$ (although showing a significant statistical standard deviation, between $20 \%$ and 50\%). Since the radius $R$ of the spheres is known, the radius of curvature $r_{1}$ was deduced from Eq. (7) with $\theta=16^{\circ}$. The radius of the capillary bridge $r_{2}$ can also be deduced by combining Eqs. (7) and (8), which shows a good agreement with the direct measurements (discrepancy of less than 6\%). Finally, a mean capillary force value was calculated for each Ucon oil mass content by statistical averaging over a dozen of individual liquid bridges, the resultant standard deviation being used as an error bar.

As shown in Fig. 4, and despite a weak decrease of the mean capillary force with $\zeta_{u}$, the obtained values can be reasonably approximated by a constant force in this range of Ucon oil mass contents, with a median value $F_{\text {cap }}=0.425 \pm 0.013 \mathrm{mN}$. It is worth noting that this force is larger but of the same order of magnitude than the buoyant weight of a single particle, $\left(\rho_{b}-\rho_{l}\right) g \pi / 6 d^{3} \approx 0.19 \mathrm{mN}$. An almost constant capillary force regardless of the liquid content is perfectly consistent with the

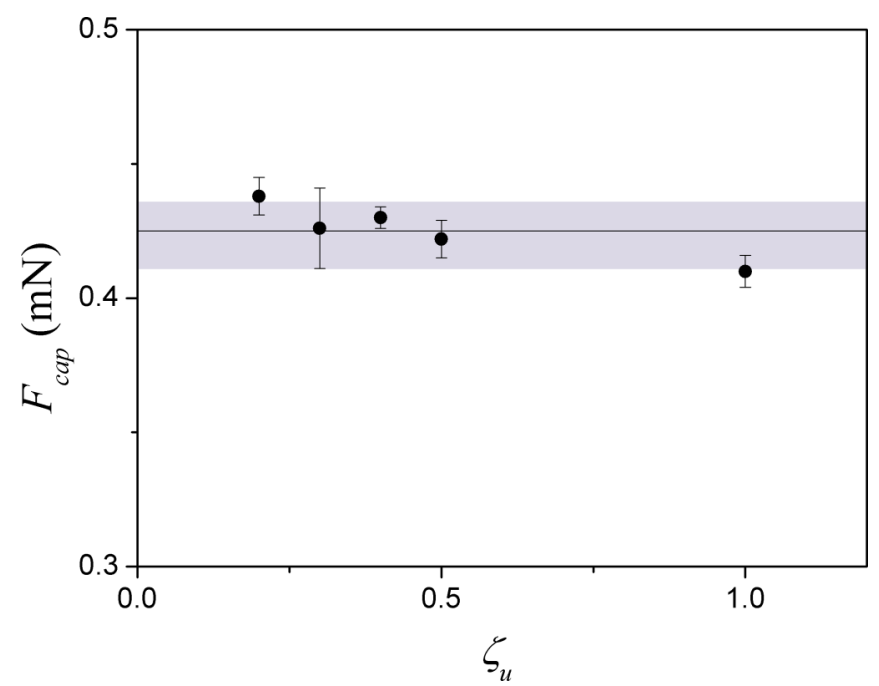

FIG. 4. Mean capillary force due to a liquid bridge of Ucon oil between two $3 \mathrm{~mm}$ borosilicate glass spheres as a function of the Ucon oil mass content $\zeta_{u}$. The mean capillary force weakly decreases with $\zeta_{u}$ but remains roughly constant in this range of Ucon oil mass content, with a median value $F_{\text {cap }}=0.425 \pm 0.013 \mathrm{mN}$. The solid line stands for the median value, while the light gray rectangle depicts the overall error domain. 
theoretical prediction: the maximum capillary force generated by a liquid bridge, which is obtained for two spheres in contact (or very close to), is indeed known to be very weakly dependent on the volume of the liquid bridge $[66,67,69]$. Naturally, this no longer applies when the distance between the two bonded spheres substantially increases, noting that the separation distance at which the liquid bridge ultimately breaks is controlled by the dimensionless liquid bridge volume [67].

As already mentioned in Sec. III A, the cohesive granular samples eventually get immersed into the RIM oil mixture for the JET erosion tests. The mean capillary forces should alternatively be measured in immersed conditions instead of in air. However, this task is very challenging, and it proved unfeasible to visualize simultaneously the liquid bridge and the bead surface, even if the Ucon oil was dyed, since there is no more refraction in such conditions. Therefore, the previous results obtained in air are assumed to be qualitatively (but not quantitatively) the same for the immersed samples, especially regarding the very weak dependence of the mean capillary force on Ucon oil mass content evidenced in Fig. 4.

\section{Solid bonds}

In contrast to the case of the liquid bridges, the shape of a solid bond connecting two beads provides no direct information about the minimal force needed to break it. For this reason, we developed two specific mechanical tests enabling the measurement of either the yield strength of an individual solid bond or the maximal stress needed to fracture a sample of cohesive granular material at a substantially larger scale. The following section presents the details of the tensile tests at both micro- and macroscales.

\section{Microscale tensile test}

The microscale tensile tests performed here consist in measuring individually the maximal extensional force sustained by a given solid bond before breakage. Several experimental devices enabling force measurement at the grain scale can be found in the literature, for either shear or tensile forces [71-75]. A rather simpler setup has been developed here, the tensile force measurement being realized as follows. First, a couple of bonded beads is carefully extracted from a sample of material prepared by the protocol described in the previous Sec. III A. Then the bond is oriented vertically while the upper bead is grabbed with a clamp that is connected to a scale via a spring. Next, a lifting plate is slowly brought into contact with the lower bead after a small drop of fast hardening glue (Super Glue 3 by Loctite) is deposited on the plate so as to fix firmly the second bead. It can be noted that the glue bonding is significantly stronger than the solid resin bond to be tested. From this starting situation, the plate is slowly moved down while the force value measured by the scale is recorded until the final rupture of the solid bond. In this respect, a very soft spring is used so that the range of necessary plate movement until bond breakage is kept in the order of several centimeters. Note also that, for practical purposes, only beads with a diameter of $3 \mathrm{~mm}$ were used. A sketch of the setup is displayed in Fig. 5(a).

Figure 5(b) shows an example of a couple of beads before and after debonding, where residual parts of the broken bond can be appreciated on the surface of both beads. This suggests a cohesive failure scenario, since the breakage seems to take place within the resin bond. It can also be noted that the solid resin is not distributed homogeneously in the solid bond: in fact, and as already mentioned, during the drying process both the solidification and evaporation lead to a very significant volume reduction that shapes a final toroidal structure of the solid bond with an empty volume around the contact zone of the beads. This type of crown-shaped solid bond is expected to be more fragile than alternative ones derived simply from solidification of capillary bridges, whose shapes are likely to be preserved due to rather small volume changes.

A typical result of a microscale tensile test is presented in Fig. 6, showing that the force recorded by the scale increases continuously as the spring gets elongated by the lowering of the lifting plate. The force ultimately drops to a final constant value that simply corresponds to the weight of the upper debonded bead. The bond tensile yield force $F_{t}^{*}$ is thus given by the difference between the 


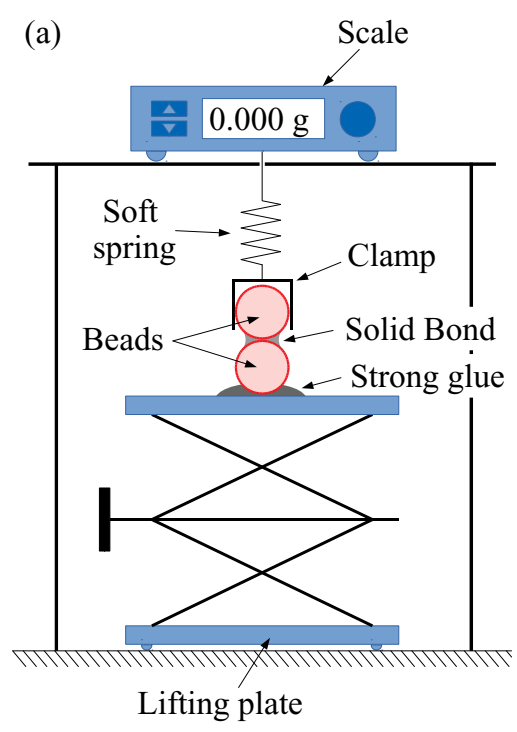

(b)
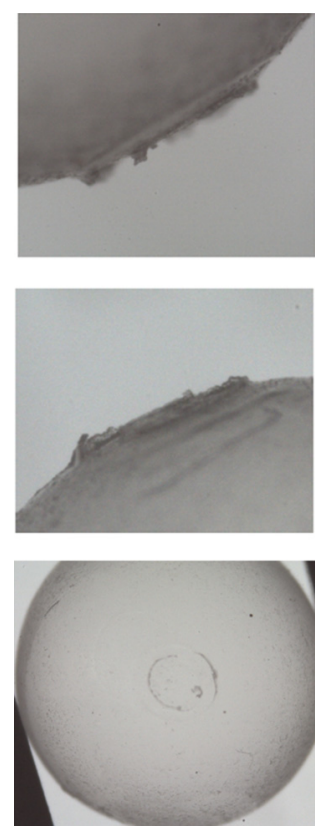

FIG. 5. (a) Sketch of the traction test setup between two bonded beads. (b) Pictures of the breakage of a solid resin bond between two borosilicate beads, featuring larger beads than those used in the actual tests for an improved visibility of the bond rupture (here $7 \mathrm{~mm}$ in diameter, just as in Fig. 2).

maximal force at breakage and the final postrupture force. As usual in mechanical rupture tests, the yield values differ significantly from one bead couple to another. Therefore, more than 15 couples of bonded beads were analyzed for each resin content sample and the results are averaged, giving a still significant but already acceptable standard deviation (see Fig. 7 where the mean tensile yield force $F_{t}^{*}$ is plotted for different initial resin mass contents $\zeta_{r}$ ). Here it can be highlighted that the

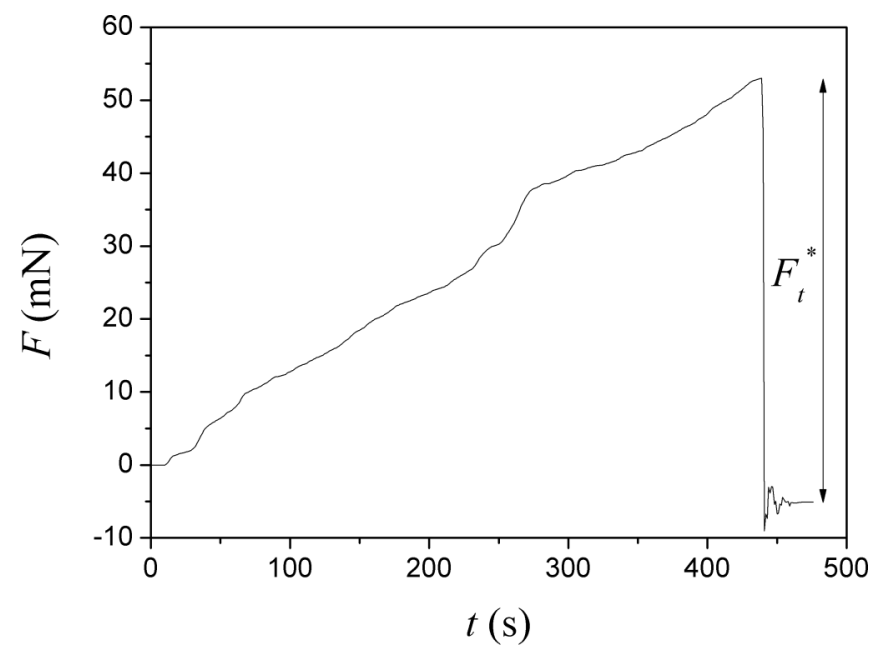

FIG. 6. Typical force measurement recorded during a test and enabling the determination of the bond tensile yield force $F_{t}^{*}$ as depicted on the curve. 


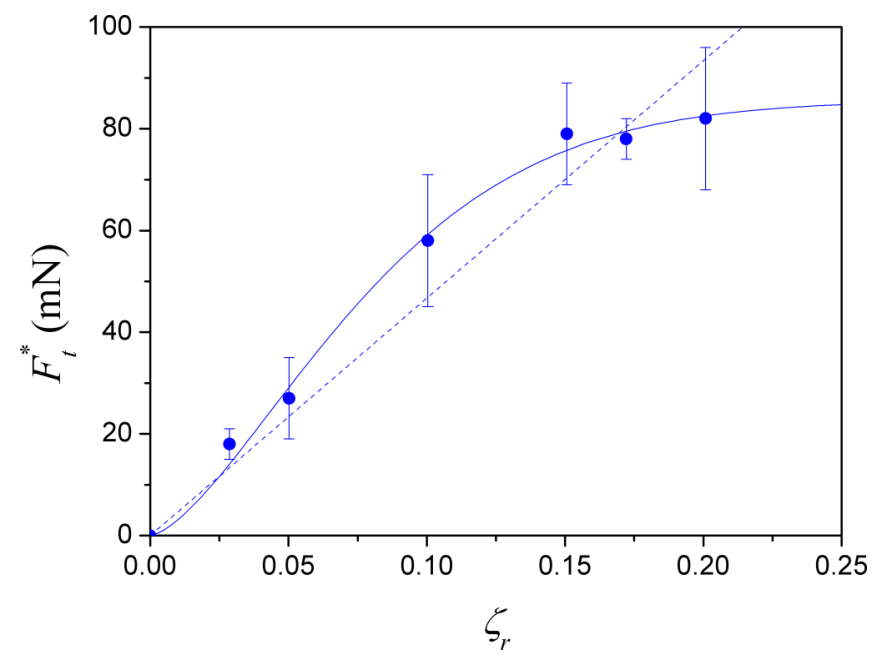

FIG. 7. Yield tensile force $F_{t}^{*}$ measured from the microscale traction test as a function of the initial resin mass content $\zeta_{r}$ for silicate beads with $2.85<d<3.30 \mathrm{~mm}$. The dashed line is a proportional relation $F_{t}^{*}=$ $A^{F} \zeta_{r}$ with $A^{F}=467 \mathrm{mN}$ (and $\zeta_{r}$ in \%) while the solid line stands for the stretched exponential law in Eq. (10) with $B^{F}=85.6 \mathrm{mN}$ and $\zeta_{r}^{F}=9.0 \times 10^{-2}$ (in percent by mass).

values obtained for the yield tensile forces are typically two orders of magnitude higher than the capillary forces created by a liquid bridge (see, for instance, Fig. 4).

The figure shows that the first values of the tensile strength increase approximately linearly from zero (cohesionless case) while the highest ones seem to almost reach a steady state around $F_{t}^{*} \simeq$ 80-90 $\mathrm{mN}$. In spite of substantial error bars due to the dispersion of the measured values, the yield tensile force may at first be roughly approximated by a linear relationship (solid line in Fig. 7)

$$
F_{t}^{*}=A^{F} \zeta_{r}
$$

with $A^{F}=467 \pm 17 \mathrm{mN}, \zeta_{r}$ the resin mass content percent by mass, and providing a coefficient of correlation $R^{2}=0.983$.

However, a more satisfying empirical relationship should predict an asymptotic plateau. The use of a simple exponential function indeed slightly increases the goodness-of-fit (i.e., $R^{2}=0.986$ ) but a greater improvement is found with a stretched exponential relation

$$
F_{t}^{*}=B^{F}\left\{1-\exp \left[-\left(\zeta_{r} / \zeta_{r}^{F}\right)^{\beta^{F}}\right]\right\} .
$$

Kept as a free parameter, the optimal value for the exponent is $\beta^{F}=1.44 \pm 0.11$. With a view to simplification and without lowering significantly the goodness-of-fit, we chose to fix $\beta^{F}=3 / 2=$ 1.5 and consequently obtained $B^{F}=85.6 \pm 1.8 \mathrm{mN}$ and $\zeta_{r}^{F}=(9.0 \pm 0.4) \times 10^{-2}$ (in percent by mass) with a coefficient of correlation $R^{2}=0.994$. This latter empirical relation, shown in dashed line in Fig. 7, will be used in the following to quantify the yield tensile force for any initial resin mass content $\zeta_{r}$.

\section{Macroscale tensile test}

The tensile resistances of our artificial cohesive materials can also be measured at a larger (sample) scale, with a more representative number of particles. To this end we use a cell made of two conical parts (see Fig. 8). The lower part is fixed on the bottom, while the upper part is initially attached with clamps to the lower one at their common (narrower) section, which is a disk of $30 \mathrm{~mm}$ in diameter. A sample of beads with a given liquid resin is first prepared aside then poured into the cell. After complete drying (several days), the fixing clamps are removed and an increasing 


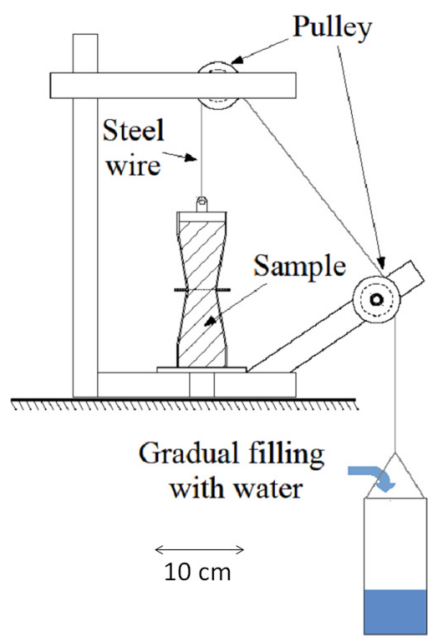

FIG. 8. Sketch of the traction device at the sample scale.

load is applied on the upper cone, via a pulley system, until ultimate separation of both parts. The resulting critical force divided by the failure section permits the estimation of a tensile yield stress, denoted $\sigma_{t}^{*}$. Due to finite size effects, only the samples constituted of the beads of diameter $1 \mathrm{~mm}$ were tested. Note that this setup is somehow reminiscent of previous ones developed to study the macroscopic cohesion of a wet granular material [76,77].

The values of $\sigma_{t}^{*}$ obtained for several initial resin mass contents are plotted in Fig. 9. The error bars were estimated by realizing three identical traction tests for similar samples with $\zeta_{r}=0.1 \%$ that gave a standard deviation around $15 \%$ and then assuming the same relative uncertainty for the other resin contents. The tendency observed in Fig. 9 is rather similar to the previous experimental results on wet granular material [76,77] and is also clearly comparable to the one of bond yield

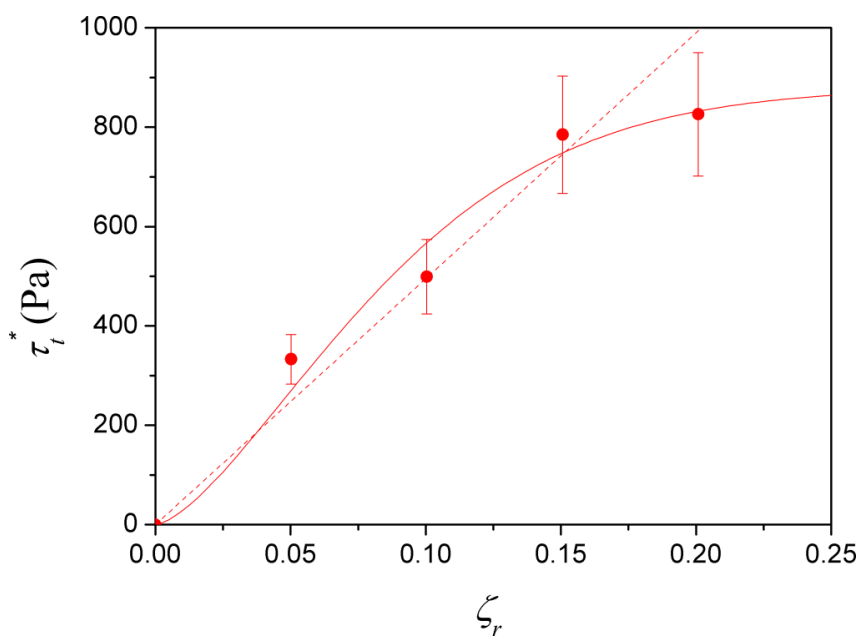

FIG. 9. Yield tensile stress $\tau_{t}^{*}$ measured from the macroscale traction test as a function of the initial resin mass content $\zeta_{r}$ for silicate beads with $0.75<d<1.0 \mathrm{~mm}$. The dashed line is a proportional relation $\sigma_{t}^{*}=$ $A^{\sigma} \zeta_{r}$ with $A^{\sigma}=4.95 \mathrm{kPa}$ (and $\zeta_{r}$ in percent) while the solid line stands for the stretched exponential relation in Eq. (12) with $B^{\sigma}=879 \mathrm{~Pa}$ and $\zeta_{r}^{\sigma}=9.8 \times 10^{-2}$ (in percent by mass). 


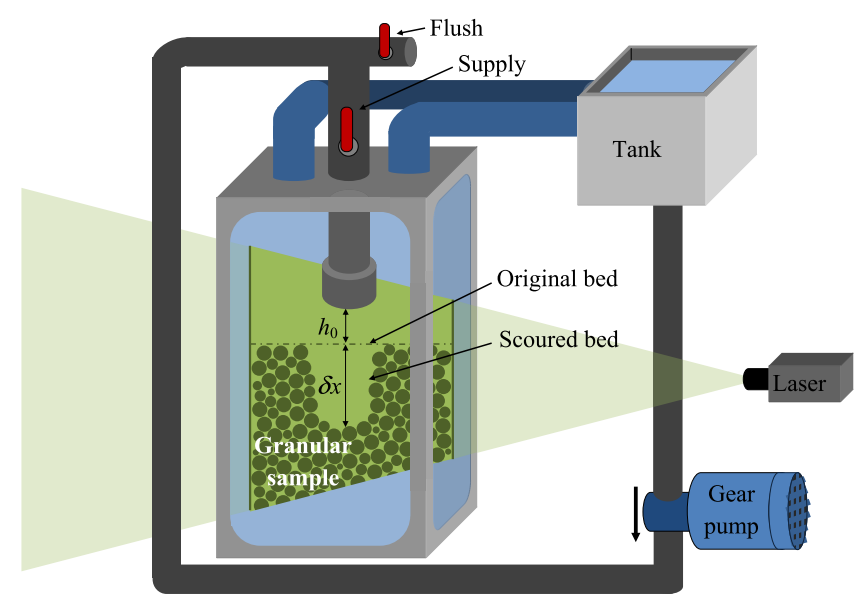

FIG. 10. Sketch of the Jet Erosion Test device adapted for refractive-index-matching visualization.

tensile strength shown in Fig. 7, with an initially linear evolution that tends to saturate at higher resin contents at a speculated asymptotic value in the range 850-900 Pa. Here again, and consistent with the empirical relations proposed for the microscale data, both a crude proportional law (solid line in Fig. 9 and a more accurate stretched exponential relation (dashed line in Fig. 9) can be proposed,

$$
\sigma_{t}^{*}=A^{\sigma} \zeta_{r}
$$

with $A^{\sigma}=4.95 \pm 0.42 \mathrm{kPa}$ and a coefficient of correlation $R^{2}=0.966$, and

$$
\sigma_{t}^{*}=B^{\sigma}\left\{1-\exp \left[-\left(\zeta_{r} / \zeta_{r}^{\sigma}\right)^{3 / 2}\right]\right\}
$$

with $B^{\sigma}=879 \pm 64 \mathrm{~Pa}, \zeta_{r}^{\sigma}=(9.8 \pm 1.3) \times 10^{-2}$ in percent by mass, and a better coefficient of correlation: $R^{2}=0.985$.

Here it can be noted first that the values obtained for $\zeta_{r}^{\sigma}$ and $\zeta_{r}^{F}$ are quite consistent and, second, that a substantial lower goodness-of-fit is found for the data obtained at the sample scale.

\section{RIM-JET tests}

The classical Jet Erosion Test involves an immersed jet impinging at the upper surface of the tested soil sample. As described in detail in Ref. [48], we slightly modified the original setup to our specific RIM restrictions (close loop supply, laser imaging from the lateral side, front image recording). This setup, sketched in Fig. 10, features a transparent rectangular container of $20 \mathrm{~cm}$ high and $10 \times 20 \mathrm{~cm}^{2}$ cross section. Using a gear pump, the RIM liquid for the jet is supplied at a variable flow rate (up to $230 \mathrm{~cm}^{3} \mathrm{~s}^{-1}$ ) from a reservoir tank to a circular nozzle of diameter $D_{J}=5 \mathrm{~mm}$ at the top of container. On the other hand, the liquid supply for the initial sample immersion can be performed from the bottom of the container through a parallel pipe network (not shown in the setup schematic of Fig. 10). Two overflow pipes at the top of the container bring the liquid back to the reservoir tank in a closed loop system.

The sample to be tested is prepared separately and beforehand, in a transparent box of height $15 \mathrm{~cm}$ and with dimensions slightly smaller than the container cross section. About $3 \mathrm{~kg}$ of material (i.e., glass beads roughly uniformly mixed with a given initial content of either resin or Ucon oil) are carefully poured into the box to obtain an almost homogeneous packing with an approximate solid volume fraction $\Phi=0.61 \pm 0.01$ (or equivalently a measure of the sample height equal to $110 \pm 2 \mathrm{~mm}$ ). In the case of the resin bonds, the box is put for several days in a warming chamber at 
$60{ }^{\circ} \mathrm{C}$ until complete drying. Then the box is carefully installed inside the container before closure of the top of the container. Afterwards, the granular sample is very slowly immersed with the RIM oil mixture supplied upwards from the bottom. Note that the bottom of the inner box is perforated with holes to permit the flow through the granular medium. Thereby, the flow rate is kept very low to avoid both the disturbance of the liquid bridges (in the case of Ucon oil bonds) and the appearance of air bubbles trapped between the glass beads. Once the sample is completely immersed, the liquid supply is switched to the upper circular jet nozzle for a downwards injection. The initial distance between the outlet of the injection nozzle and the surface of the granular sample is kept approximately at about $h_{0} \simeq 70 \mathrm{~mm}$.

By projecting a planar laser from the lateral side (Coherent, PL $532 \mathrm{~nm}, 100 \mathrm{~mW}$, fan angle $60^{\circ}$ ), the vertical plane of the sample containing the jet axis can be visualized by the fluorescent dye added to the RIM oil mixture (Red Nile, Fluka). Additionally, a high-pass optical filter (Thorlabs, $580 \mathrm{~nm}$ ) is used to filter the reflected and refracted lights. The jet erosion process is recorded from the front window with a digital camera (model MQ042MG-CM supplied by Ximea, pixel resolution of $2048 \times 2048$ ), typically at a frame rate of $1 \mathrm{fps}$ and up to $50 \mathrm{fps}$ in some specific cases. The pixel resolution of the images is about $9 \mathrm{px} \mathrm{mm}^{-1}$.

\section{JET EROSION OF COHESIVE GRANULAR MEDIA}

\section{A. Experimental protocols}

With our RIM-JET device, both the erosion and scouring process are then controlled by varying the jet flow rate $Q_{J}$ and the corresponding mean jet velocity $U_{J}=\frac{4 Q_{J}}{\pi D_{J}^{2}}$. Note that the jet Reynolds number which reads $\operatorname{Re}_{J}=\rho_{l} \frac{U_{J} D_{J}}{\mu_{l}}$ remains below 1770. Considering that the dimensionless jet length $h_{0} / D_{J}$ is always less than 15 , the comprehensive experimental study of submerged jets by Lemanov and coworkers [78] appears to suggests that all our experiments lie within the laminar regime, the transition to turbulence being only observed at a greater distance from the jet outlet.

As detailed just below (see also Ref. [48]), two complementary protocols are used to determine either the critical conditions for erosion initiation or the subsequent scouring kinetics through crater expansion.

\section{Increasing jet velocity protocol: Erosion onset}

The first protocol was developed to assess the jet conditions for which some grains are first carried away by the impinging flow. To this end, the pump flow rate is continuously increased from zero with a constant rate of $7 \mathrm{~cm}^{3} \mathrm{~s}^{-1}$ per minute. Using a synchronized timer, the frame sequence generally recorded at $1 \mathrm{fps}$ enables one to identify, by difference between two successive frames, the critical flow rate $Q_{J}^{*}$, the critical jet velocity $U_{J}^{*}$, and the critical jet Reynolds number $\operatorname{Re}_{J}^{*}$ corresponding to the onset of grain motion. Note that the uncertainty on the exact time at erosion onset corresponds to a period estimated to less than $3 \mathrm{~s}$, thus leading to uncertainties of around $0.02 \mathrm{~ms}^{-1}$ and 3 for $U_{J}^{*}$ and $\mathrm{Re}_{J}^{*}$, respectively.

Concerning the materials used for this protocol, only the solid (resin) bonds were employed. In this respect, and as discussed in Sec. V below, the viscous behavior of the capillary bridges of Ucon oil strongly depends on the application time of the hydrodynamic stress, which is not suitable for the present protocol. Nevertheless, a set of preliminary tests appeared to suggest a critical jet velocity $U_{J}^{*}$ for the erosion onset that does not exceed $1 \mathrm{~m} \mathrm{~s}^{-1}$ for all liquid mass contents in Ucon oil. Concerning the solid particles themselves, both borosilicate and silica glass bead samples were tested, even if the latter ones do not allow for RIM visualization. In this case, only the upper surface of the sample is recorded by the camera, which is consequently positioned higher and inclined with an approximate angle of $45^{\circ}$, whereas the motion detection from difference between two successive frames is kept. 


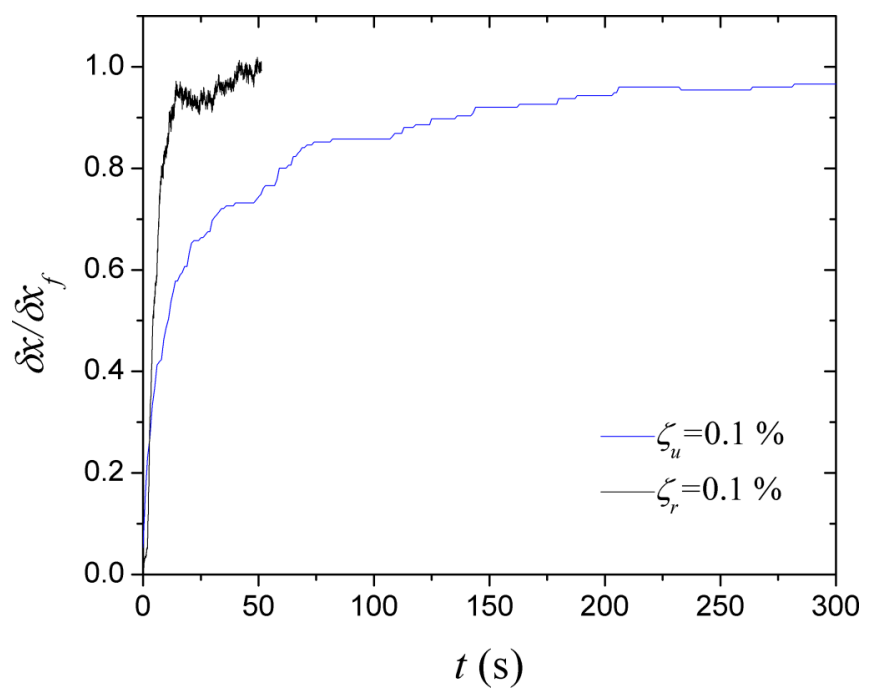

FIG. 11. Time evolution of the nondimensional crater depth $\frac{\delta x}{\delta x_{f}}$ during a JET test with an injection velocity $U_{J}=8.2 \mathrm{~m} \mathrm{~s}^{-1}$ for borosilicate glass beads $d=3 \pm 0.02 \mathrm{~mm}$ mixed with an initial resin mass content $\zeta_{r}=$ $0.1 \%$ (in black) and $U_{J}=1.5 \mathrm{~m} \mathrm{~s}^{-1}$ for the same beads mixed with Ucon oil at the same liquid mass content $\zeta_{u}=0.1 \%$ (in blue).

\section{Constant jet velocity protocol: Scour kinetics}

When the stress exerted by the impinging jet exceeds the critical condition for the erosion onset previously identified, a scouring crater develops until reaching a final equilibrium depth when erosion ultimately ceases. The kinetics of the scouring process, from the flat sample surface to the stabilized crater, is here analyzed with a second protocol which relies exclusively on the RIM and PLIF techniques. Therefore, only the samples made of borosilicate glass beads (diameter $3 \pm 0.02 \mathrm{~mm}$ ) were studied, with either viscous (Ucon oil) or solid (resin) bonds.

In practice, the test is carried out as follows: For the resin bonds, and after previous identification of the critical injection velocity $U_{J}^{*}$ with the first protocol, a new sample is prepared with identical parameters and tested under a constant jet flow rate beyond the onset value, namely, with an injection velocity $U_{J}>U_{J}^{*}$. For the case of the Ucon oil bonds, only a fixed injection velocity equal to $U_{J}=$ $1.5 \mathrm{~m} \mathrm{~s}^{-1}$ was used, consistent with the critical velocities $U_{J}^{*} \leqslant 1 \mathrm{~m} \mathrm{~s}^{-1}$ observed in this case.

The measurement of the crater depth is carried out manually after digitally enhancing the contrast of the picture. Note in this respect that some of the fluidized beads often remain inside the crater once eroded, so that the bottom of the crater was just identified and picked manually by eye for each time frame. For this task, no alternative automated procedure was found to discriminate correctly the separation between the sample and the cavity.

Figure 11 shows the typical evolution of the nondimensional crater depth $\frac{\delta x}{\delta x_{f}}$ for both the viscous (Ucon oil) and solid (resin) bonds, where $\delta x_{f}$ represents the final crater depth. The samples for these two exemplary cases were prepared with the same initial liquid mass content (i.e., $0.1 \%$ in both resin and Ucon oil) and then scoured under substantially different injection velocities, namely, at $U_{J}=8.2 \mathrm{~m} \mathrm{~s}^{-1}$ for resin bonds and $U_{J}=1.5 \mathrm{~m} \mathrm{~s}^{-1}$ for Ucon oil bonds, leading to final crater depths of approximately $\delta x_{f} \sim 121 \mathrm{~mm}$ and $\delta x_{f} \sim 58 \mathrm{~mm}$, respectively. Consistently, the corresponding critical velocities estimated from the first protocol are $U_{J}^{*} \approx 4.0 \mathrm{~m} \mathrm{~s}^{-1}$ and $U_{J}^{*} \leqslant 1 \mathrm{~m} \mathrm{~s}^{-1}$, for resin and Ucon oil bonds, respectively. A first comparison between the two curves suggests that cohesion by solid bonds requires a higher jet velocity to be eroded, but the final crater depth is reached substantially faster than with the viscous cohesion. 

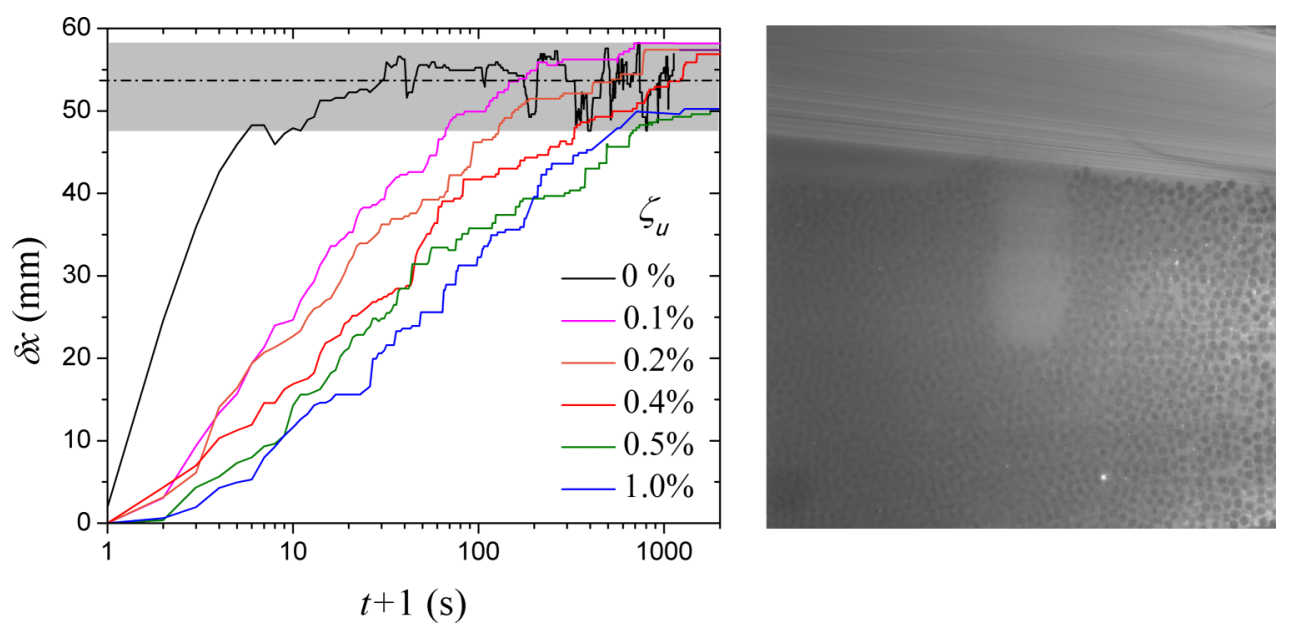

FIG. 12. Evolution of the crater depth $\delta x$ in a logarithmic timescale for different samples of borosilicate beads $\left(d=3 \pm 0.02 \mathrm{~mm}\right.$ ), with five varying Ucon oil mass contents $\zeta_{u}$, tested by RIM-JET with an injection velocity $U_{J}=1.5 \mathrm{~m} \mathrm{~s}^{-1}$. A $t+1$ timescale is used in order to plot the initial state $\delta x=0$ on the graph. The mean final crater depth obtained for a similar sample without cohesion $\left(\delta x_{f}=53.6 \mathrm{~mm}\right)$ is also represented with a dashed line, while the gray area marks the uncertainty range due to fluctuations. The picture is an illustration obtained for $\zeta_{u}=0.2 \%$.

\section{B. Cohesion by liquid bonds}

As will be discussed hereafter, the granular materials with viscous bonds cannot be satisfactorily tested with the first protocol. So only the second protocol is used, focusing specifically on the development of the scour crater when the hydrodynamic stress generated by the impinging flow remains constant, exceeding the threshold value (see Sec. IIIC). Five different samples were prepared, made of borosilicate beads $(d=3 \pm 0.02 \mathrm{~mm})$ homogeneously mixed with an increasing Ucon oil mass content: $\zeta_{u}=0.1 \%, 0.2 \%, 0.4 \%, 0.5 \%$, and $1 \%$. All the samples were then submitted to a JET test with a constant injection velocity $U_{J}=1.5 \mathrm{~m} \mathrm{~s}^{-1}$ which, from preliminary analyses, substantially exceeds the erosion onset. The evolution with time of the scour crater depth $\delta x$ is presented in Fig. 12.

For comparison, the result obtained without viscous bonds $\left(\zeta_{u}=0\right)$ in the same hydrodynamic conditions is also plotted. As already mentioned for such cohesionless JET tests [48], substantial fluctuations of the crater depth are observed in Fig. 12. Once the final crater depth is reached, the uncertainty domain (shown in gray) ranges from $\delta x_{f}=47.5 \mathrm{~mm}$ to $\delta x_{f}=58.3 \mathrm{~mm}$ with a mean value around $\delta x_{f}=54 \mathrm{~mm}$. These fluctuations are mainly due to the continuous motion existing within the crater, even after the equilibrium state is reached, with almost steady avalanches that constantly refill the crater with grains while the most exposed beads are successively swept up by the flow from the bottom of the crater. In contrast, the samples with Ucon oil bonds exhibit far less agitation due to cohesion. After rupture of their viscous bonds, the eroded beads are indeed either expelled out of the crater or pressed against the lateral wall where potentially new bonds can be created. In particular, the final equilibrium state is almost motionless, and much less fluctuation is found for the crater depth measurement.

When plotting the dimensionless final crater depths $\delta x_{f} / d$ (with $d$ the bead diameter) in Fig. 13(a), it appears that the final crater depths, both with and without cohesion, are reasonably close to each other. A slow decrease of $\delta x_{f}$ is observed when increasing the Ucon oil mass content $\zeta_{u}$. However, the maximal variation is no more than three bead diameters which, by comparison, remains within the uncertainty domain of the cohesionless case [see Fig. 12 and error bars for $\zeta_{u}=0$ in Fig. 13(a)]. As a consequence, even if more tests would be needed to conclude definitively, the 

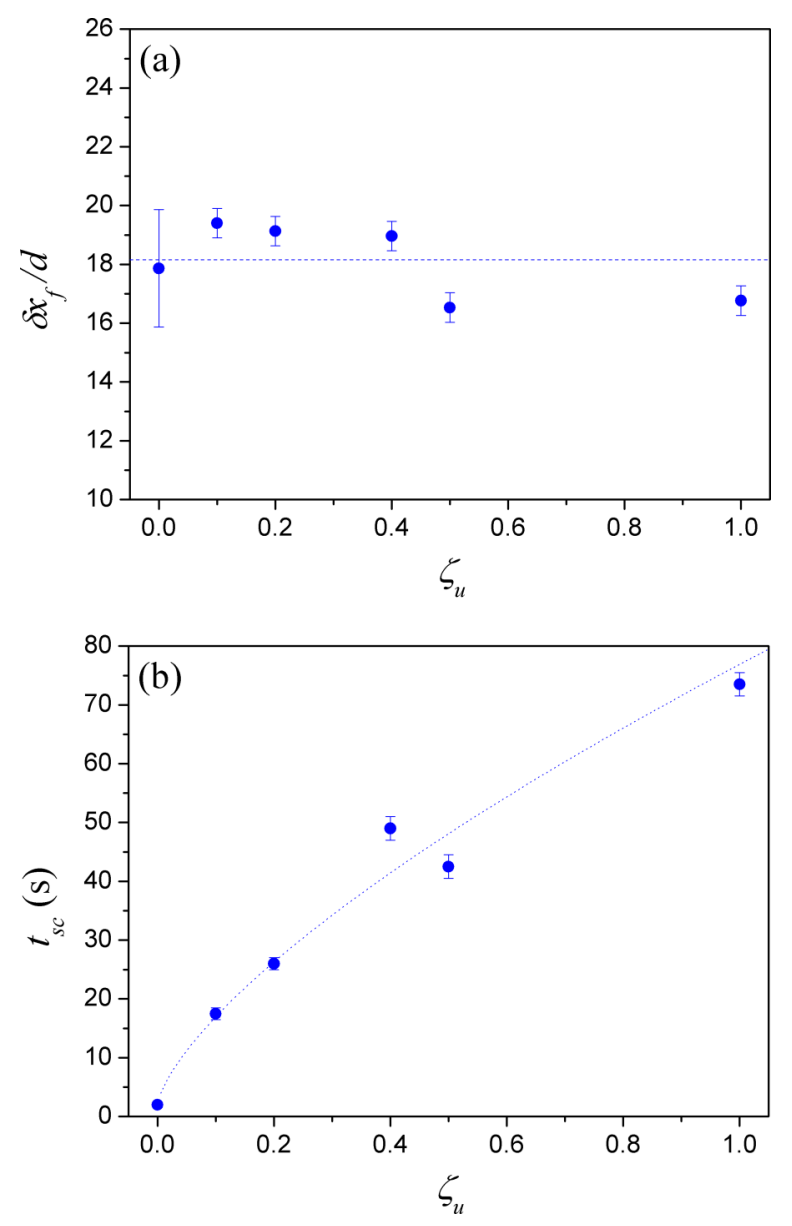

FIG. 13. (a) Final crater depth $\delta x_{f}$ normalized by the bead diameter $d$ and plotted as a function of the Ucon oil mass content $\zeta_{u}$. The error bar of the test without cohesion represents the dispersion of $\delta x_{f}$, and the one of the cohesive samples represents one bead diameter. The dashed line stands for the mean value of $\delta x_{f}$ with viscous bonds, $\delta x_{e}=54.5 \mathrm{~mm}$. (b) Characteristic scouring time $t_{s c}$ vs Ucon oil mass content $\zeta_{u}$, where $t_{s c}$ is defined as the time needed to reach $60 \%$ of the final crater depth. The dashed line is only a guide for the eye emphasizing the increase of $t_{s c}$ with $\zeta_{u}$.

final depth of the crater scoured by the impinging jet seems to be only weakly dependant on the initial mass content in Ucon oil. This result is quite consistent with the typical variation of the mean capillary force of a Ucon oil bridge according to the quantity of viscous matrix in the system, which proves to be also a very weakly decreasing function of the Ucon oil content as shown in Fig. 4. By contrast, one would not necessarily expect the cohesionless sample to reach the same final crater depth, and this could simply be a coincidence. It remains nevertheless questionable to compare both equilibrium states, since the viscous bonds induce an almost static final crater without bead movements while the absence of cohesion leads to the steady-state situation previously described, with intense grain motion and related fluctuations.

A second important trend can be inferred from Fig. 13(b) where a characteristic scouring time $t_{s c}$ (defined here as the time needed to reach $60 \%$ of the final crater depth) is plotted versus the Ucon oil mass content $\zeta_{u}$. In contrast to the final crater depth, $t_{s c}$ exhibits a clear dependence on $\zeta_{u}$, increasing continuously with the Ucon oil content and, consequently, with the average size and volume of 
the viscous bridges. This suggests that the diameter of the viscous bond controls, under almost steady stress conditions, the time needed for the liquid bridge to be stretched until ultimate breakup. Consistently, the variation of the capillary force with the distance between two equal-size spheres is known to depend substantially on the capillary bridge volume and the critical separation distance scales as a power $1 / 3$ of the capillary bridge volume [67]. As a consequence, the time required to separate two initially bonded particles will be presumably longer for a larger liquid bridge volume $[69,70]$. It is, however, not trivial to relate the initial Ucon oil mass content to the average bond diameter after complete mixing and even less to propose a direct relation between $t_{s c}$ and $\zeta_{u}$. Note in addition that viscous dissipation plays also a major role during the bridge stretching dynamics (see, for instance, Ref. [69]) and that, despite the fact that we did not test this dependence in the present experiments, shorter characteristic times can be expected for less viscous bonding liquids.

Furthermore, a last observation with very relevant implications must be also highlighted here: Despite the high viscosity of the capillary binder, the images recorded during a RIM-JET experiment reveal that a small quantity of Ucon oil seems to be washed away when exposed to the jet flow (see, for instance the black rising plume on the upper right area of the picture in Fig. 12). Upon close inspection of the RIM-PLIF images, it is indeed possible to discern roughly a kind of wash-out front that precedes the subsequent scouring development. Only a rather small fraction of Ucon oil appears to be entrained by the interstitial flow since substantial cohesion remains at the end of a test, as particularly evidenced by the stability of an almost vertical wall of the final scoured crater. The loss of Ucon oil is thus likely to be taking place both at the edge of the liquid bridges and at the beads' surface, while it also appears to decrease gradually with the distance from the impingement point. Here it is worth noting that in previous experiments by Lick and coworkers [41], where the erosion rates were determined as a function of shear stress for uniform millimetric quartz particles with added bentonite, almost similar observations were made: the small bentonite particles, agglomerated as a paste, did not bind properly the quartz particles and were progressively removed from the bed during the tests.

This established fact bears two important implications. First, the partial wash-out of the capillary bridges precludes any accurate control of the cohesion by initial Ucon oil content within our model systems. Second, the cohesive strength within the sample evolves in both time and space, making it very complicated to estimate the onset conditions for erosion, especially with our first protocol that gives rise to a rather long exposure time before reaching the initiation of erosion. For these reasons, our study of erosion of granular materials with viscous cohesive bonds will remain limited to the previous qualitative interpretation, obtained only with our second protocol, without any further experiments or more systematic analysis.

\section{Cohesion by solid bonds}

In contrast to the liquid bridges, the solid bonds remain relatively unaffected by the surrounding fluid flow until they eventually break under excessive hydrodynamic stress. Thus, both protocols can be used to determine, first, the critical flow conditions for erosion, and, second, to analyze the kinetics of scouring above the previous threshold.

Based on the experimental results obtained with the increasing jet velocity protocol, the critical jet injection velocity $U_{J}^{*}$ needed to remove a particle is plotted in Fig. 14 as a function of the initial resin mass content $\zeta_{r}$ for the two types of beads with different sizes.

From the comparison between the silica and borosilicate glass beads of the same size about $d \approx 3 \mathrm{~mm}$, we can first note that only a very weak and almost negligible gap is observed in the results, actually of the same order of magnitude as the inherent dispersion of the data. The small difference of density between silica glass $\left(\rho_{s}=2,495 \mathrm{~kg} \mathrm{~m}^{-3}\right)$ and borosilicate glass $\left(\rho_{b}=2,230\right.$ $\mathrm{kg} \mathrm{m}^{-3}$ ) affects little, if at all, their erosion, suggesting that the contribution of both buoyant weight and solid friction to the resistance against erosion is here negligible compared to that of cohesion. For a smaller bead diameter, $0.75 \mathrm{~mm}<d<1.0 \mathrm{~mm}$, a substantial decrease of $U_{J}^{*}$ is found, which means that smaller particles are less resistant for a same initial resin content. Regarding the general 


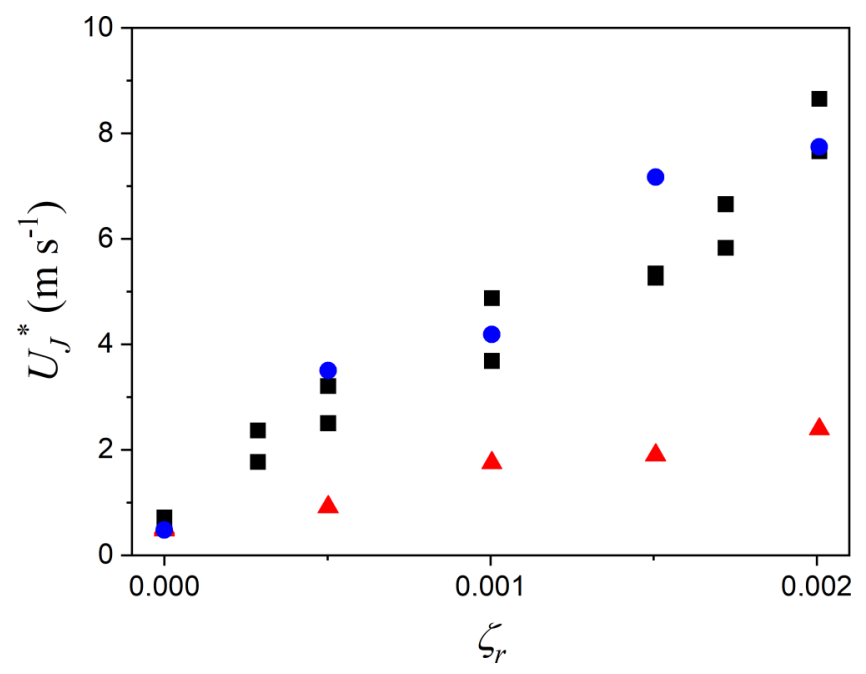

FIG. 14. Critical injection velocity $U_{J}^{*}$ as a function of the initial resin mass content $\zeta_{r}$ for borosilicate beads with $d=3 \pm 0.02 \mathrm{~mm}(\bullet)$ and silicate beads with $2.85<d<3.30 \mathrm{~mm}(\boldsymbol{\square})$ and $0.75<d<1.0 \mathrm{~mm}$ $(\boldsymbol{\Lambda})$. Error bars are not shown here since they are smaller than the markers' size.

tendency, the critical velocity $U_{J}^{*}$ systematically tends to increase monotonically with the initial resin mass content $\zeta_{r}$.

A quantitative analysis can be carried out by plotting the critical value of the maximal shear stress $\tau_{m}^{*}$ at the sample surface based on previous Eq. (5). This is shown in Fig. 15 using a log$\log$ representation, where a power law with an exponent $3 / 2$ can satisfactorily approximate the experimental data for the increase of $\tau_{m}^{*}$ with $\zeta_{r}$ [see Fig. 15(a)]. The coefficients of correlation are $R^{2}=0.982,0.914$, and 0.950 for borosilicate beads with $d=3 \pm 0.02 \mathrm{~mm}$, and silicate beads with $2.85<d<3.30 \mathrm{~mm}$ and $0.75<d<1.0 \mathrm{~mm}$, respectively. The corresponding slopes seem to be mainly ruled by the particles' diameter since similar values are obtained for $d \approx 3 \mathrm{~mm}$, while a substantially smaller slope fits the data for $d \approx 1 \mathrm{~mm}$. This dependence with $d$ appears compatible with a simple quadratic relationship, thus giving rise to the following empirical relation : $\tau_{m}^{*} \propto$ $\zeta_{r}{ }^{3 / 2} d^{2}$, as evidenced in Fig. 15(b), with a still acceptable goodness-of-fit $R^{2}=0.905$. However, we have for the moment no physical interpretation capable to explain such a scaling for the cohesive strength induced by solid bonds, especially for our particular system where the final resin bridges are crown-shaped due to large volume reduction after complete drying (see Sec. III B 2).

Considering now some impinging flow conditions where the previous shear-stress threshold is exceeded, the progression of erosion and the gradual development of a scour crater have been followed due to our second protocol. Figure 16 shows exemplarily the evolution of the crater depth $\delta x$ for a sample made of borosilicate glass beads (diameter $3 \pm 0.02 \mathrm{~mm}$ ) with an initial resin mass content $\zeta_{r}=0.2 \%$ and for the following successive increments in jet velocity: 9.45, 9.63, 9.80, 9.96, and $10.16 \mathrm{~m} \mathrm{~s}^{-1}$.

As shown in Fig. 16, the scouring starts for an injection velocity of $U_{J}=9.63 \mathrm{~m} \mathrm{~s}^{-1}$. In particular, and as revealed by the images recorded during the test, two aggregates containing a few number of particles were here expelled by the flow, corresponding to a maximal excavation of about one particle diameter high. This scouring phase lasts less than $2 \mathrm{~s}$. Then no more erosion occurs during about $40 \mathrm{~s}$, despite two successive incremental increases of the velocity, $U_{J}=9.80$ and $9.96 \mathrm{~m} \mathrm{~s}^{-1}$. Eventually, and after being exposed for approximately $7 \mathrm{~s}$ to a new incremental velocity $U_{J}=10.16 \mathrm{~m} \mathrm{~s}^{-1}$, the sample gets eroded again but quite vigorously this time. A sharp increase of the crater depth is then observed before the scouring progressively slows down until reaching a final depth of $\delta x_{f} \simeq 32 d \simeq 95 \mathrm{~mm}$. 

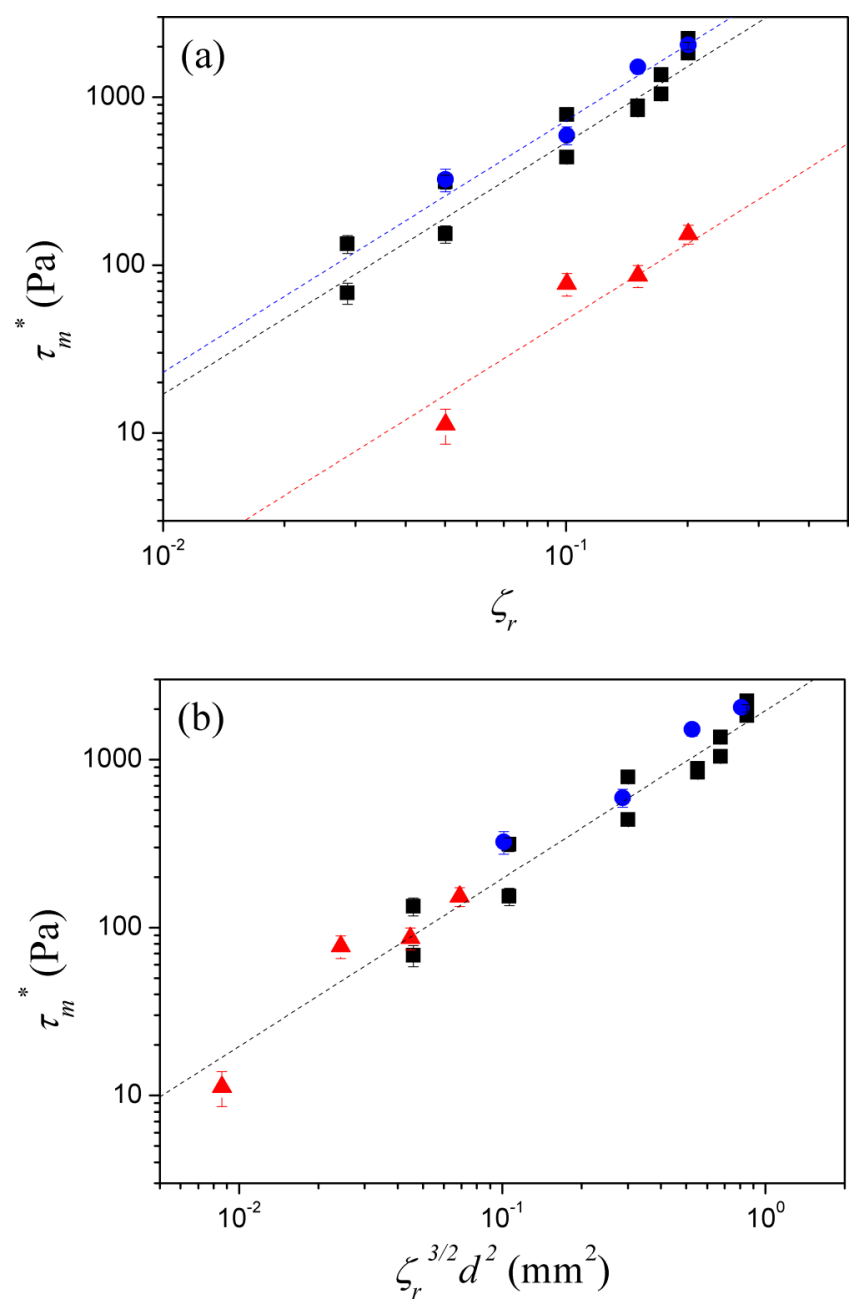

FIG. 15. Critical shear stress $\tau_{m}^{*}$ exerted by the impinging flow at erosion onset as a function of (a) the initial resin mass content $\zeta_{r}$ and (b) the combination $\zeta_{r}^{3 / 2} d^{2}$, for borosilicate beads with $d=3 \pm 0.02 \mathrm{~mm}$ $(\bullet)$ and silicate beads with $2.85<d<3.30 \mathrm{~mm}(\boldsymbol{\square})$ and $0.75<d<1.0 \mathrm{~mm}(\boldsymbol{\Delta})$. The dashed lines stand for power-law relationships: $\tau_{m}^{*} \propto \zeta_{r}^{3 / 2}$ (a) and $\tau_{m}^{*} \propto \zeta_{r}^{3 / 2} d^{2}$ (b).

Figure 17 shows four pictures extracted from the image sequence recorded during the test presented in Fig. 16 and highlighting the final crater excavation observed at $U_{J}=10.16 \mathrm{~m} \mathrm{~s}^{-1}$ in Fig. 16. More precisely, and adopting a new time origin at the moment where the jet velocity was increased from $9.96 \mathrm{~m} \mathrm{~s}^{-1}$ to $U_{J}=10.16 \mathrm{~m} \mathrm{~s}^{-1}$, the images (a)-(d) were successively taken at incremental times $\delta t=7 \mathrm{~s}, 8 \mathrm{~s}, 14 \mathrm{~s}$, and $41 \mathrm{~s}$, respectively. The corresponding dimensionless crater depths are approximately $\delta x / d=3,10,21$, and 32 .

Unlike the cohesionless case where a small increase in jet velocity would induce a rather limited crater depth increase without any time delay [48], a certain exposure time is needed here to induce erosion but, once the scouring process is sufficiently initiated, its kinetics are rather abrupt, with a very large final depth considering the rather moderate change in hydrodynamic stress. By means of several image sequences recorded at a substantially higher frame rate, it was possible to assess the role of the eroded particles in the sudden crater formation and thus to understand this difference of behavior compared to the scouring of cohesionless soils. In fact, it appears that most of the eroded 


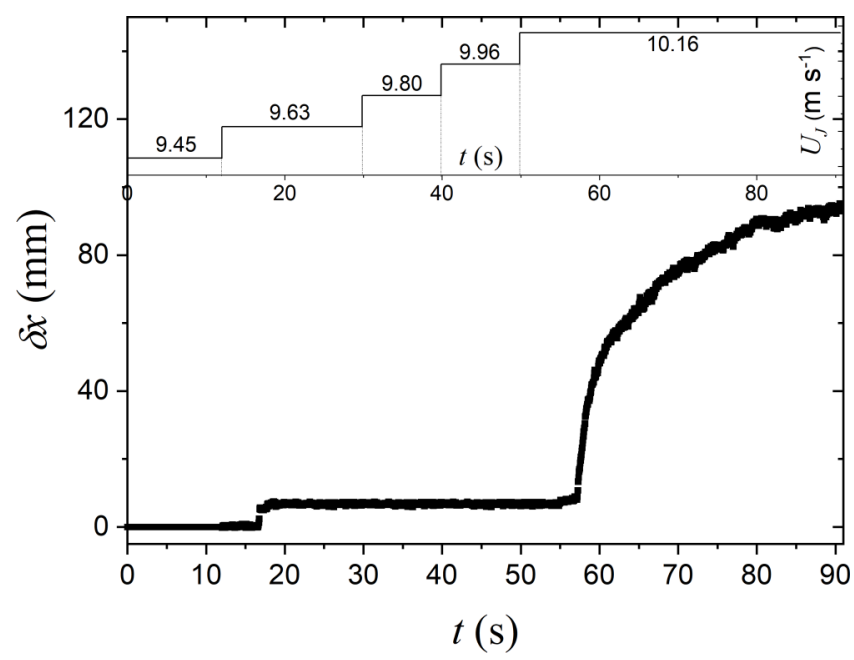

FIG. 16. Time evolution of the crater depth $\delta x$ within a sample of borosilicate glass beads (diameter $3 \pm$ $0.02 \mathrm{~mm}$ ) with an initial resin mass content $\zeta_{r}=0.2 \%$. Successive incremental steps of injection velocity $U_{J}$ are implemented, from $9.45 \mathrm{~m} \mathrm{~s}^{-1}$ to $10.16 \mathrm{~m} \mathrm{~s}^{-1}$.
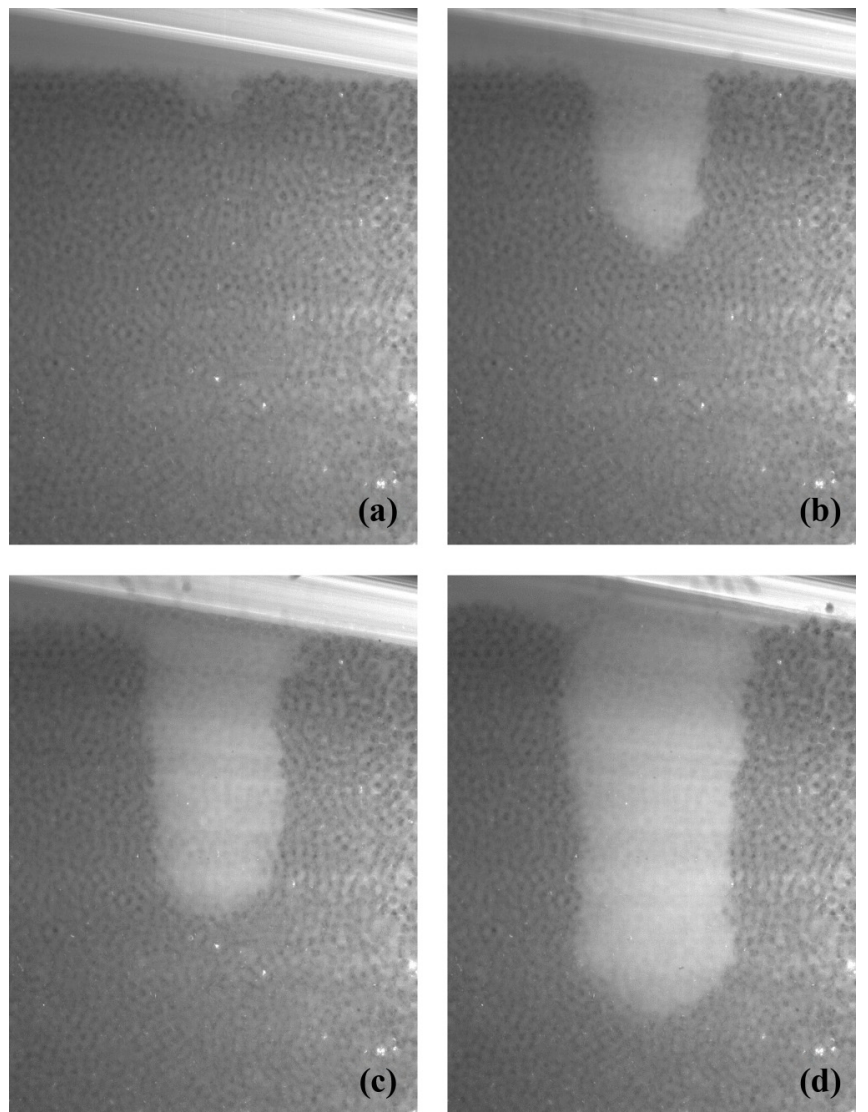

FIG. 17. JET test on the sample of borosilicate glass beads (diameter $3 \pm 0.02 \mathrm{~mm}$ ) with an initial resin mass content $\zeta_{r}=0.2 \%$, with an injection velocity $U_{J}=10.16 \mathrm{~m} \mathrm{~s}^{-1}$. The images in the sequence are taken $7 \mathrm{~s}(\mathrm{a}), 8 \mathrm{~s} \mathrm{(b)}, 14 \mathrm{~s}$ (c), and $41 \mathrm{~s}$ (d) after the increment of velocity. 


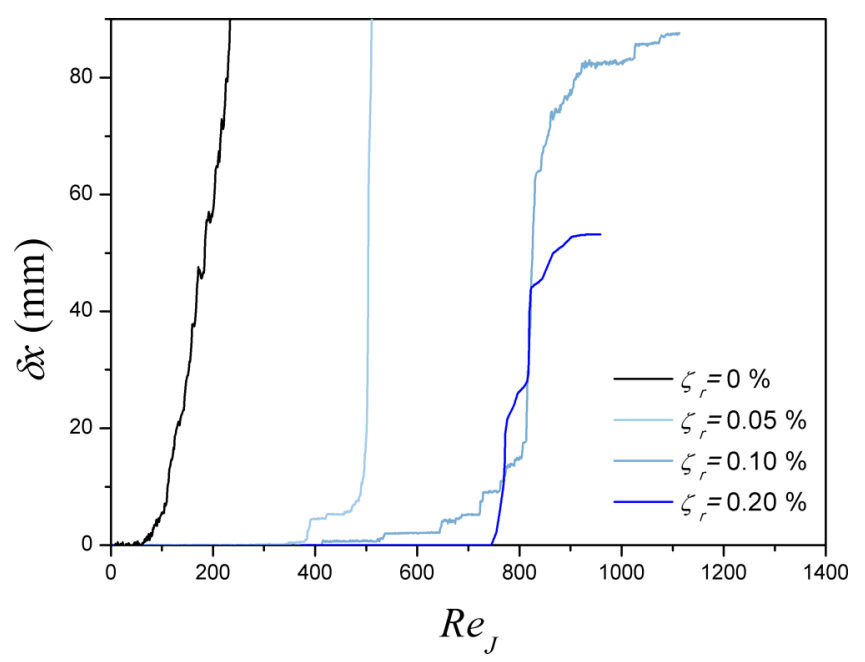

FIG. 18. Evolution of the crater depth $\delta x$ during a time-linear increase of the jet velocity and plotted versus the instantaneous jet Reynolds number $\mathrm{Re}_{j}$. The tests were carried out with samples of borosilicate glass beads (diameter $3 \pm 0.02 \mathrm{~mm}$ ) without cohesion (black curve), $\zeta_{r}=0 \%$, and with initial resin mass contents $\zeta_{r}=0.05 \%$ (light blue curve), $\zeta_{r}=0.10 \%$ (blue curve), and $\zeta_{r}=0.20 \%$ (dark blue curve).

particles get trapped in a convective cell within the crater. After being ejected upwards along the lateral wall of the scoured hole, many of these particles are drawn back downwards by the impinging jet and so get caught in a rather steady vortex. The presence of those particles in the suspension seems to enhance considerably the erosion at the impinging zone due to the additional, and presumably efficient, contribution of the interparticle collisions to the breakage of solid bonds. Thus, the crater development accelerates as the concentration of eroded/suspended particles increases. However, as the scour depth quickly grows, the hydrodynamic stress progressively decreases and thus mitigates the erosion until a new equilibrium is ultimately reached. This scenario requires that such a convective cell of suspended grains takes place in the crater, suggesting that a kind of critical depth has been reached. Note that this is somehow reminiscent to a previous numerical study about soil erosion by an axisymmetric impinging jet flow where an instability of the jet, i.e., the emergence of a reversed flow in the crater depth, was observed when the scour depth exceeds $\delta_{x} \sim 20 \mathrm{~mm}$ [55].

The comparison with the cohesionless situation can be made clearer by use of the first protocol where the injection velocity, and consequently the related jet Reynolds number $\mathrm{Re}_{j}$, increase linearly with time. In this respect, Fig. 18 shows the evolution of the crater depth $\delta x$ with the instantaneous jet Reynolds number $\operatorname{Re}_{j}$ for samples made of borosilicate beads $(d=3 \mathrm{~mm})$ with the following initial resin mass contents: $\zeta_{r}=0$ (cohesionless case), $\zeta_{r}=0.05 \%, \zeta_{r}=0.10 \%$, and $\zeta_{r}=0.20 \%$, respectively. Note that the range in $\delta x$ is displayed only up to $90 \mathrm{~mm}$. Beyond that limit the results are significantly disturbed by the presence of the sample's lower boundary.

Figure 18 confirms the very substantial increase with resin content of the sample's erosion threshold, expressed here through the critical jet Reynolds number at which the scouring is initiated. Regarding specifically the difference between cohesive and cohesionless cases, it clearly appears that, without cohesion, a progressive and almost linear evolution of the scour depth is observed, following rather steadily the linear increase of the jet Reynolds number. In contrast, the erosion of the cohesive samples is a far more abrupt process, with sudden scouring phases often interrupted by intermediate periods of low (if any) erosion. Furthermore, the erosion and scouring are far less intense when cohesion (i.e., the resin content) increases, in terms both of the instantaneous erosion rate and of the overall scour depth. 
To conclude this section it appears relevant to comment on the reproducibility of the obtained results despite the limited number of experiments performed so far. While the tests showed a rather satisfactory repeatability concerning the erosion threshold, the subsequent scour development appears more random, whereby two systematic patterns seem to emerge nevertheless. First, as already explained in the description of Fig. 16, the scouring kinetics very often begins with a succession of small and rapid erosion bursts, interspersed with motionless periods lasting from few to tens of seconds. Second, as soon as the crater is deep enough, the evolution is always observed to become continuous with no longer intermittence.

\section{FURTHER ANALYSIS OF EROSION ONSET WITH COHESION}

\section{A. Definition of a cohesion number Co}

In surface erosion of soils, where individual particles are removed from the sediment bed by the hydrodynamic forces generated by a superficial fluid flow, the presence of cohesion or adhesion in between neighboring particles can substantially increase the soil's resistance to erosion, in addition to buoyant weight and related friction force which are the only resistant forces for cohesionless materials. As already proposed by several authors [43-45], a dimensionless group can be constructed to quantify the respective contribution of cohesion and gravitational force. This cohesion number Co, defined as the ratio of cohesion/adhesion to buoyant weight, can be written either at a local scale, regarding the forces acting on an individual soil particle, or at a macroscopic scale, in terms of stresses within a representative elementary volume of soil.

In the present study, the buoyant weight can be easily expressed as a force or a stress, namely, $F_{g} \propto\left(\rho_{b}-\rho_{l}\right) g d^{3}$ or $\sigma_{g} \propto\left(\rho_{b}-\rho_{l}\right) g d$ where $d$ is the bead diameter and $\rho_{b}$ and $\rho_{l}$ are the densities of beads and liquid, respectively. On the other hand, a theoretical expression for cohesive or adhesive force and stress is far less straightforward; see, e.g., Refs. [25,26,39,41,42]. Therefore we adopted here an experimental estimation from the yield tensile force $F_{t}^{*}$ and stress $\tau_{t}^{*}$ values, measured at either the micro- and macroscale as detailed in Sec. III B 2. Then, and omitting prefactors, two alternative definitions can be used for the cohesion number Co, comparing either $F_{t}^{*}$ to $F_{g}$ or $\tau_{t}^{*}$ to $\sigma_{g}$. The latter expression was adopted here and a remaining issue consists in relating the microscopic yield tensile force $F_{t}^{*}$ to the macroscopic yield tensile stress $\tau_{t}^{*}$. To this end, we employ the following formula initially proposed by Rumpf [79]:

$$
\tau_{t}^{*}=\frac{k \Phi}{\pi} \frac{F_{t}^{*}}{d^{2}}
$$

where $k$ stands for the coordination number while $\Phi$ is the solid volume fraction.

Note that it was not possible to test this relation with our experimental data because it was not achievable to measure in parallel $F_{t}^{*}$ and $\tau_{t}^{*}$ for a same bead diameter. This is partly so because the microscale manipulation is hardly possible for spheres smaller than 2 or $3 \mathrm{~mm}$ and on the other hand because finite-size effects might be predominant at the sample scale when the ratio of the cell size to the particle diameter becomes too small.

Several laws exist to relate the coordination number $k$ to the solid volume fraction $\Phi$ as $k=\pi /(1-\Phi)$ [79] or $k=2 \sqrt{3} \Phi /(1-\Phi)$ [80], which give, respectively, $k \approx 8.06$ and $k \approx 5.42$ for $\Phi=0.61$ as evaluated in our experiments. However, due to substantial dispersion on $k$, we preferred to rely on previous results by Aste and coworkers [81] obtained from the x-ray microtomography technique in disordered monosize sphere packings, very similar to our own samples. By interpolation of Aste's data obtained for six different values of solid volume fraction, one gets $k \approx 6.40$ for $\Phi=0.61$, giving rise to the following relation between $\tau_{t}^{*}$ and $F_{t}^{*}$ :

$$
\tau_{t}^{*} \approx 1.25 \frac{F_{t}^{*}}{d^{2}}
$$


On this basis, a unique definition of Co can be used as follows:

$$
\mathrm{Co}=\frac{\tau_{t}^{*}}{\left(\rho_{b}-\rho_{l}\right) g d}=\frac{1.25 F_{t}^{*}}{\left(\rho_{b}-\rho_{l}\right) g d^{3}} .
$$

As an example, the buoyant weight of the $3 \mathrm{~mm}$ borosilicate glass beads is approximately $0.19 \mathrm{mN}$, which is considerably smaller than the values of $F_{t}^{*}$ obtained in Fig. 7, giving rise to cohesion numbers up to about 300 . By comparison, the cohesion number for the same beads with capillary bridges of Ucon oil is only slightly larger than 2 .

\section{B. Proposal of a generalized Shields number}

As discussed before, a proper definition of a dimensionless group able to account for critical conditions at the erosion onset for a cohesive granular material must necessarily include cohesive and/or adhesive stresses in addition to friction and gravitational contributions. This means that the traditional expression of the Shields number $\mathrm{Sh}_{0}$ in Eq. (4) no longer applies and must be improved. To this end, we propose a straightforward refinement by adding the cohesion/adhesion to the buoyant weight in Eq. (4) and assuming this additional contribution to be directly proportional to the macroscopic yield tensile stress $\tau_{t}^{*}$. Then the following generalized Shields number Sh can be proposed, with $\alpha$ an empirical coefficient, expectedly of order 1, to be more precisely determined as

$$
\mathrm{Sh}=\frac{\tau_{m}}{\left(\rho_{b}-\rho_{l}\right) g d+\alpha \tau_{t}^{*}} .
$$

Introducing then Eqs. (4) and (15), one gets

$$
\mathrm{Sh}=\frac{\mathrm{Sh}_{0}}{1+\alpha \mathrm{Co}} .
$$

The relevance of this proposal is tested in Fig. 19 as a function of the particle Reynolds number, showing the experimental values of the critical Shields number $\mathrm{Sh}_{0}^{*}$ based on the usual definition in Eq. (4) for cohesionless sediments, from the previous data presented in Fig. 15 and using Eq. (5) to express the critical fluid shear stress at erosion onset. Obviously, $\mathrm{Sh}_{0}^{*}$ is no longer appropriate in presence of cohesion/adhesion, as evidenced by a systematic and substantial increase over up to three orders of magnitude. Incidentally, such a drastic increase of the classical Shields number is commonly observed for fine sediments such as formalized qualitatively by Coleman and Nikora [23] through the internal attractive stresses generated by aggregation, cohesion, or adhesion between particles, respectively.

Then, relying on the proposed definition of a generalized Shields number Sh in Eq. (17), the related critical values Sh* are also plotted in Fig. 19(a) with open markers. By comparison with both the Shields curve and the previous cohesionless data by Ref. [48], the corresponding agreement is relatively satisfactory. Prior to this, it was necessary to estimate both the cohesion number Co and the empirical coefficient $\alpha$, which was done in the following manner: First, the cohesion number Co was evaluated from Eq. (15) using estimated values of either the yield tensile force $F_{t}{ }^{*}$ [based on the extrapolation law given in Eq. (10) for both the borosilicate and silicate beads with a diameter of $3 \mathrm{~mm}$ ] or the yield tensile stress $\tau_{t}^{*}$ [based on the extrapolation law given in Eq. (12) for the silicate beads with $0.75<d<1.0 \mathrm{~mm}$ ]. As shown in Fig. 19(b), the unknown coefficient $\alpha$ was adjusted for the whole data by linear regression according to the following relation between $\mathrm{Sh}_{0}^{*}$, $\mathrm{Sh}^{*}$, and Co: $\mathrm{Sh}_{0}^{*} / \mathrm{Sh}^{*}-1=\alpha \mathrm{Co}$. The corresponding optimal value is $\alpha=2.26 \pm 0.27$ with a coefficient of correlation $R^{2}=0.813$.

To conclude, the following comments can be made: First, it is worth noting that $\alpha_{\text {opt }}$ is reasonably close to unity, as expected from a dimensional analysis to define the cohesion number Co. However, considering exclusively the tensile resistance of a solid bond is undoubtedly an oversimplification and, ideally, the respective contributions of resistant shear and torque should also be included in the analysis. Second, it can be observed that the data obtained for Sh* in Fig. 19 are substantially scattered. This scattering is most probably caused by uncertainties in the measurement from both 

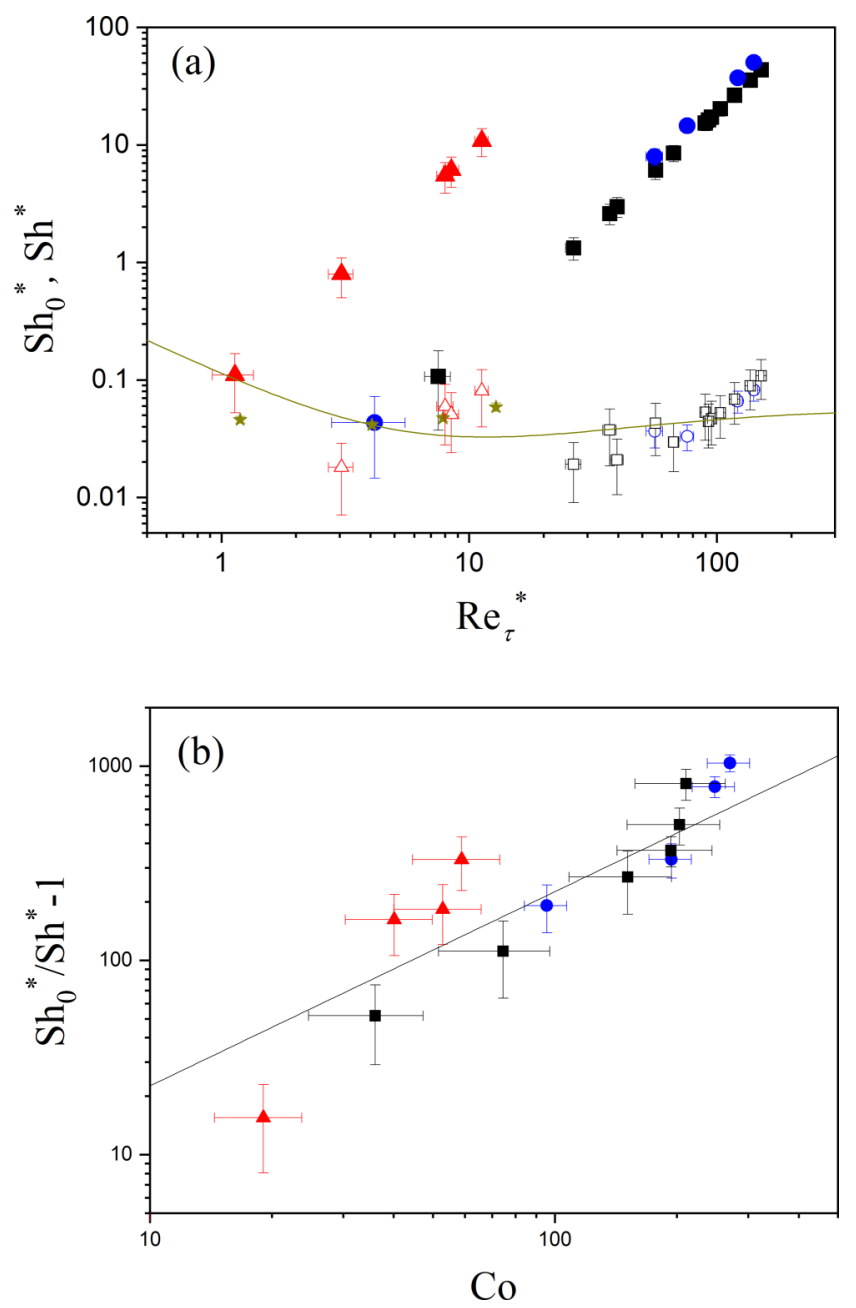

FIG. 19. (a) Critical values at erosion onset of the classical Shields number $\mathrm{Sh}_{0}^{*}$ (closed symbols) and the generalized Shields number Sh* (open symbols), with $\alpha=2.26$ [see Eq. (17)], obtained for borosilicate beads with $d=3 \pm 0.02 \mathrm{~mm}$ (blue circles) and silicate beads with $2.85<d<3.30 \mathrm{~mm}$ (black squares) and $0.75<d<1.0 \mathrm{~mm}$ (red triangles). The cohesionless values extracted from Ref. [48] (dark yellow stars) and the implicit formulation of the Shields curve by Guo [18] (solid line) are also shown for comparison. (b) Plot of $\left(\mathrm{Sh}_{0}^{*} / \mathrm{Sh}^{*}-1\right)$ versus the cohesion number Co for all the data with cohesion. The line stands for a linear regression with a slope $\alpha=2.26\left(R^{2}=0.813\right)$.

the traction and erosion tests, and also from the use of several empirical laws (namely, in evaluating $F_{t}^{*}, \tau_{t}^{*}$, and $\tau_{m}$ ) that is not entirely satisfactory. However, even if a substantial dispersion is found, the data based on the generalized Shields number are obviously brought significantly and consistently closer to the Shields curve compared to the usual definition, thus providing a substantial improvement for the modeling of erosion and transport processes of natural sediments.

\section{CONCLUSION}

The experimental campaign and analytical process to derive a generalized formulation of the Shields criterion for weakly cohesive materials have been described in this paper. This includes 
the development of artificial granular materials for the test samples featuring a certain degree of intergranular cohesion/adhesion provided by either liquid or solid bonds. The elementary capillary force of the liquid bridges has been estimated directly by means of graphical analysis of the bonds' geometry and well-known analytical expressions. On the other hand, the cohesive strength of the solid bonds has been evaluated by measuring both the yield tensile force and stress, respectively, at the particle and sample scales with specific traction tests built for this purpose.

Concerning the surface erosion tests, a device with the typical JET configuration has been adapted for optical RIM conditions to permit a direct visualization of the scouring process. The tests themselves were performed following two different experimental protocols addressing the erosion onset and the scouring kinetics, respectively. To this end, the hydrodynamic interpretation model for the erosion onset has been revisited based on the numerical results recently produced by Benseghier and coworkers [49], providing an enhanced estimation of the maximum shear stress at the impingement zone.

Several valuable insights on the surface erosion of cohesive materials have been derived based on the presented experimental results:

(1) The cohesion provided by the liquid (capillary) bridges was found to feature an almost constant strength irrespective of their mass fraction, which appears to be consistent with the observation of a similar final scour depth for all the corresponding tests. Nevertheless, the initial mass fraction of the capillary liquid appears in fact to play a relevant role on the erosion kinetics, leading to a substantial increase of the characteristic scouring time, which is most probably related to viscous dissipation effects at the integranular contacts. In any case, the fact that the hydrodynamic seepage effects seemed to induce a partial wash-out of the cohesive binder during the tests highlights the inherent difficulties for a quantitative analysis of the tests with capillary cohesion.

(2) Concerning the samples with solid adhesive bonds, the tests have evidenced the complexity of the yielding process. The significant volume reduction of the cohesive matrix during the hardening process of the solid bonds appears to be largely responsible for their irregular (quasitoroidal) shape and a key factor for their yield strength. The latter has been measured mechanically in terms of the resin mass content, qualitatively suggesting the existence of a certain saturation threshold and asymptotic behavior, for which a quantitative empirical relation has been proposed. Consistent with these observations, the critical value of the maximal shear stress during the JET tests appears to be well described by a power law of the resin mass content with an exponent of $3 / 2$ and a quadratic dependence on the particle size. As expected, the classical form of the Shields number is no longer suitable to describe the erosion onset and the scouring kinetics were also strongly modified in presence of the solid cohesion, with sudden bursts of erosion being observed repeatedly during the tests.

(3) Finally, a generalized Shields expression accounting for the cohesion/adhesion stress in addition to the buoyant weight and friction has been put forward along with a complementary definition of a Cohesion number. This generalized Shields number manages to rationalize and reconcile in a single graph the present data with the so-called Shields curve and previous data of authors with cohesionless materials.

This study opens up several research prospects in the short and medium terms. First, and from the experimental point of view, it appears very relevant to enlarge the data basis with further additional tests involving resin bonding and above all to broaden the scope to other types of materials to extend the generality of the present results. This seems especially important in view of the practical difficulties, both for testing and for interpretation, arising from the high volume reduction of the resin bridges mentioned before. In this sense, an ongoing project by the authors is now starting to investigate specifically some alternative cohesive systems with the following main goals: (1) to test Rumpf's relationship between yield tensile force and stress, (2) to analyze the occurrence of finite size effects in tensile tests at sample scale, and (3) to extend the mechanical characterization of cohesion including complementary shear tests along with the presented tensile tests. From the theoretical perspective, a forthcoming article is under preparation featuring the numerical micromechanical model (LBM-DEM approaches) recently developed by Benseghier [49] 
and focusing, among other topics, on the relevance of the generalized Shields expression proposed here to describe the results of 2D JET simulations on granular soils with solid cohesion.

\section{ACKNOWLEDGMENTS}

The authors acknowledge financial support from the région Sud, Provence-Alpes-Côte d'Azur (France), and valuable exchanges with D. Chaussée (Suez Consulting Company). This work has benefited from fruitful discussions with P. Aussillous, J.-Y. Delenne, and Y. Khidas. The contribution of Jean-Loup Sarrat as an internship student is also acknowledged.

\section{APPENDIX}

As explained in the main text, the recent numerical results by Benseghier et al. [49] for 2D impinging jet in laminar regime have suggested the adequacy of a Blasius-like friction law for the maximal bed shear stress $\tau_{m}$ and can be consistently adapted to real 3D jet flows (injection diameter $D_{J}$, injection mean velocity $U_{J}$ ), thus providing the following expression for $\tau_{m}$ :

$$
\tau_{m}=a \operatorname{Re}_{J}^{-1 / 2} \rho_{l}\left[\widetilde{u}_{3 D}\left(h_{0}\right)\right]^{2},
$$

where $a$ is a constant to be fixed, $\operatorname{Re}_{J}$ is the jet Reynolds, $\operatorname{Re}_{J}=\frac{\rho_{l} U_{J} D_{J}}{\mu_{l}}$, and $\tilde{u}_{3 D}\left(h_{0}\right)$ stands for the maximal vertical velocity predicted from a classical self-similar model for laminar free round jet [54]. This velocity is evaluated at a distance $h_{0}$ from the jet outlet, accounting for the previously mentioned virtual origin $\lambda$, which is the source point from which the jet appears to emerge when observed sufficiently far from the outlet [59]. According to Brunier-Coulin and coworkers [48], the following empirical expression $\lambda \approx 0.048 \operatorname{Re}_{J} D_{J}$ can be used for a laminar round jet. Finally, the maximal velocity $\widetilde{u}_{3 D}\left(h_{0}\right)$ from the laminar self-similar model reads

$$
\widetilde{u}_{3 D}\left(h_{0}\right)=U_{J} \frac{\operatorname{Re}_{J}}{8} \frac{D_{J}}{h_{0}+\lambda}=\frac{\mu_{l}}{\rho_{l}} \frac{\operatorname{Re}_{J}^{2}}{8\left(h_{0}+\lambda\right)} .
$$

In contrast to Refs. [48] or [53], a complete definition of the cohesionless Shields number can be used now, $\mathrm{Sh}_{0}=\frac{\tau_{m}}{\left(\rho_{g}-\rho_{l}\right) g d}$, with $\rho_{g}$ the grain density being equal to either $\rho_{b}$ or $\rho_{s}$ for borosilicate and silicate glass beads, respectively. Additionally, the approximate particle Reynolds number $\operatorname{Re}_{p}=\frac{\rho_{l} \widetilde{u}_{3} d}{\mu_{l}}$ previously used by these authors can be advantageously replaced by the shear Reynolds number $\operatorname{Re}_{\tau}$, which is indeed the correct dimensionless number to be used in the classical Shields approach, defined by

$$
\operatorname{Re}_{\tau}=\frac{\rho_{l} u_{\tau} d}{\mu_{l}}
$$

with the friction velocity $u_{\tau}$ being given by

$$
u_{\tau}=\sqrt{\frac{\tau_{m}}{\rho_{l}}} .
$$

Note that, according to Eq. (A1), the two previous Reynolds numbers are simply related by $\operatorname{Re}_{\tau}=a^{1 / 2} \operatorname{Re}_{J}^{-1 / 4} \operatorname{Re}_{p}$. Note also that, after introduction of the Archimedes number defined by $\mathrm{Ar}=\frac{\rho_{l}\left(\rho_{g}-\rho_{l}\right) d^{3}}{\mu_{l}{ }^{2}}$, the critical Shields number $\mathrm{Sh}_{0}^{*}$ is simply related to the shear Reynolds number $\operatorname{Re}_{\tau}$ by

$$
\mathrm{Sh}_{0}^{*}=\operatorname{ArRe}_{\tau}^{2} .
$$

Based on these completed definitions of Shields and Reynolds numbers, Brunier-Coulin's dataset will be used, following the same methodology [48], to set the most accurate value for the coefficient $a$. To this end, and assuming that the onset of erosion is reached for a critical Shields number value 
TABLE II. Results from Ref. [48] obtained for different bead diameters $d$ with the corresponding values of Archimedes number $\mathrm{Ar}$, ratio $\mathrm{Sh}_{0}^{*} / a$ of cohesionless Shields number to friction law coefficient and mean particle Reynolds number $\left\langle\operatorname{Re}_{p}^{*}\right\rangle$.

\begin{tabular}{lccccc}
\hline \hline$d(\mathrm{~mm})$ & $\mathrm{Ar}$ & $\mathrm{Sh}_{0}^{*} / a$ & $R^{2}$ & $\mathrm{Re}_{\tau}$ & $\mathrm{Sh}_{0}^{*}(\mathrm{Guo})$ \\
\hline 1 & 14.7 & $0.153 \pm 0.012$ & 0.962 & 1.19 & 0.0977 \\
3 & 396 & $0.138 \pm 0.010$ & 0.969 & 4.05 & 0.0414 \\
5 & 1832 & $0.158 \pm 0.011$ & 0.979 & 7.85 & 0.0336 \\
7 & 5028 & $0.196 \pm 0.017$ & 0.940 & 12.83 & 0.0327 \\
\hline \hline
\end{tabular}

$\mathrm{Sh}_{0}^{*}$, Eq. (A1) can be inserted in the expression of $\mathrm{Sh}_{0}^{*}$ giving

$$
\mathrm{Sh}_{0}^{*}=a \frac{\mu_{l}^{2}}{\rho_{l}\left(\rho_{g}-\rho_{l}\right) g d^{3}} \operatorname{Re}_{J}^{* 7 / 2}\left[\frac{d}{8\left(h_{0}-\lambda\right)}\right]^{2} .
$$

Finally, after some calculation, the following power-law relation is found between the critical jet Reynolds number $\operatorname{Re}_{J}^{*}$ and the distance $h_{0}$ from the jet outlet to the sample's upper surface:

$$
\operatorname{Re}_{J}^{* 7 / 4}=8\left(\frac{\mathrm{Sh}_{0}^{*}}{a} \mathrm{Ar}\right)^{1 / 2} \frac{h_{0}+\lambda}{d} .
$$

Compared to the previous analysis by Brunier-Coulin and coauthors [48], the exponent of the power law changed from 2 to $7 / 4$ due to the addition of the Blasius-type friction coefficient.

Then, owing to Eq. (A7), a linear regression of $\mathrm{Re}_{J}^{* 7 / 4}$ versus $h_{0}+\lambda$ can satisfactorily provide a value of $\mathrm{Sh}_{0}^{*} / a$ for each particle diameter, $d=1,3,5$, and $7 \mathrm{~mm}$, with a slightly smaller but fully comparable goodness-of-fit. The corresponding values, including coefficient of correlation $R^{2}$, are listed here above, in Table II.

The coefficient $a$ in the friction law remains at this stage unknown. It needs to be tuned in order to agree with the Shields curve given in Ref. [14] when the critical Shields number $\mathrm{Sh}_{0}^{*}$ is plotted versus the shear Reynolds number $\operatorname{Re}_{\tau}$. More conveniently, we use here the following explicit formulation

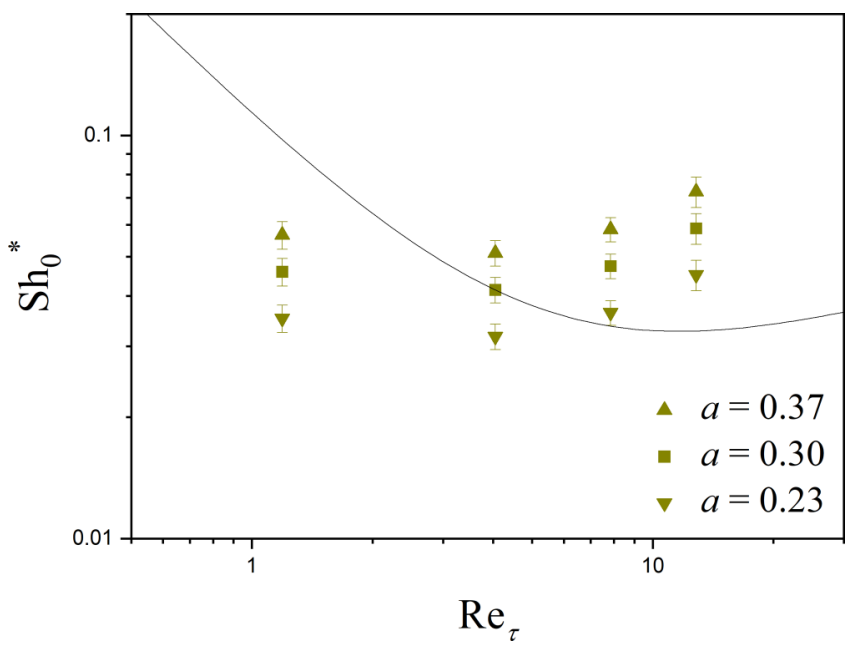

FIG. 20. Critical Shields number $\mathrm{Sh}_{0}^{*}$ versus shear particle Reynolds number $\operatorname{Re}_{\tau}$ for $a=0.30$ (squares), $a=0.37$ (up triangles), and $a=0.23$ (down triangles). The solid line stands for the Shields curve given by the explicit formulation from Guo [18]. 
of the Shields curve from Guo [18]:

$$
\mathrm{Sh}_{0}^{*}=\frac{0.106}{\operatorname{Re}_{\tau}}+0.0545\left[1-\exp \left(-0.158 \mathrm{Re}_{\tau}^{0.52}\right)\right]
$$

Combining this relation with previous Eq. (A5), a pair of values for $\mathrm{Re}_{\tau}$ and $\mathrm{Sh}_{0}^{*}$ is calculated for each particle diameter as reported in Table II. Then a coefficient $a=0.30 \pm 0.07$ is obtained analytically by minimization of the mean-squared error when comparing the experimental values of $\mathrm{Sh}_{0}^{*}$ with their related theoretical values from Guo's formulation (see Fig. 20).

[1] K. Zhang, B. C. Douglas, and S. P. Leatherman, Global warming and coastal erosion, Clim. Change 64, 41 (2004).

[2] O. Evrard, J. P. Laceby, H. Lepage, Y. Onda, O. Cerdan, and S. Ayrau, Radiocesium transfer from hillslopes to the Pacific Ocean after the Fukushima Nuclear Power Plant accident: A review, J. Environ. Radioact. 148, 92 (2015).

[3] L. C. van Rijn, Principles of Sediment Transport in Rivers, Estuaries and Coastal Seas (Aqua Publications, Amsterdam, 1993).

[4] J. C. Winterwerp and W. G. van Kesteren, Introduction to the Physics of Cohesive Sediment Dynamics in the Marine Environment, Developments in Sedimentology Vol. 56 (Elsevier, Amsterdam, The Netherlands, 2004).

[5] M. A. Nearing, L. D. Norton, D. A. Bulgakov, G. A. Larionov, L. T. West, and K. M. Dontsova, Hydraulics and erosion in eroding rills, Water Resour. Res. 33, 865 (1997).

[6] C. Castillo and J. A. Gómez, A century of gully erosion research: Urgency, complexity and study approaches, Earth-Sci. Rev. 160, 300 (2016).

[7] K. Van Oost, T. A. Quine, G. Govers, S. De Gryze, J. Six, J. W. Harden, J. C. Ritchie, G. W. McCarty, G. Heckrath, J. V. Giraldez, J. R. Marques da Silva, and R. Merckx, The impact of agricultural soil erosion on the global carbon cycle, Science 318, 626 (2007).

[8] L. J. Prendergast and K. Gavin, A review of bridge scour monitoring techniques, J. Rock Mech. Geotech. Eng. 6, 138 (2014).

[9] E. Bollaert and A. Schleiss, Scour of rock due to the impact of plunging high velocity jets Part I: A state-of-the-art review, J. Hydraul. Res. 41, 451 (2003).

[10] S. Bonelli (ed.), Erosion of Geomaterials (Wiley-ISTE, London, 2012).

[11] S. Bonelli (ed.), Erosion in Geomechanics Applied to Dams and Levees (John Wiley \& Sons, New York, 2013).

[12] M. Foster, R. Fell, and M. Spannagle, The statistics of embankment dam failures and accidents, Can. Geotech. J. 37, 1000 (2000).

[13] K. S. Richards and K. R. Reddy, Critical appraisal of piping phenomena in earth dams, B. Eng. Geol. Environ. 66, 381 (2007).

[14] J. M. Buffington, The legend of A. F. Shields, J. Hydraul. Eng. 125, 376 (1999).

[15] S. Dey, Entrainment threshold of loose boundary streams, in Experimental Methods in Hydraulic Research, edited by P. Rowinski (Springer, Berlin, 2011), p. 2948.

[16] A. Hong, M. Tao, and A. Kudrolli, Onset of erosion of a granular bed in a channel driven by fluid flow, Phys. Fluids 27, 013301 (2015).

[17] B. Allen and A. Kudrolli, Granular bed consolidation, creep, and armoring under subcritical fluid flow, Phys. Rev. Fluids 3, 074305 (2018).

[18] J. Guo, Discussion of “The Albert Shields Story” by Junke Guo, J. Hydraul. Eng. 123, 666 (1997).

[19] Z. Cao, G. Pender, and J. Meng, Explicit formulation of the Shields diagram for incipient motion of sediment, J. Hydraul. Eng. 132, 1097 (2006).

[20] H. Lee and S. Balachandar, Critical shear stress for incipient motion of a particle on a rough bed, J. Geophys. Res. 117, F01026 (2012). 
[21] A. Kudrolli, D. Scheff, and B. Allen, Critical shear rate and torque stability condition for a particle resting on a surface in a fluid flow, J. Fluid Mech. 808, 397 (2016).

[22] B. R. Sutherland and S. Dalziel, Bedload transport by a vertical jet impinging upon sediments, Phys. Fluids 26, 035103 (2014).

[23] S. E. Coleman and V. I. Nikora, A unifying framework for particle entrainment, Water Resour. Res. 44, W04415 (2008).

[24] W. Jacobs, P. Le Hir, W. van Kesteren, and P. Cann, Erosion threshold of sandmud mixtures, Cont. Shelf Res. 31, S14 (2011).

[25] D. Chen, Y. Wang, B. Melville, H. Huang, and W. Zhang, Unified formula for critical shear stress for erosion of sand, mud, and sand-mud mixtures, J. Hydraul. Eng. 144, 04018046 (2018).

[26] M. Righetti and C. Lucarelli, May the Shields theory be extended to cohesive and adhesive benthic sediments? J. Geophys. Res. 112, C05039 (2007).

[27] T. J. Tolhurst, K. S. Black, and D. M. Paterson, Muddy sediment erosion: Insights from field studies, J. Hydraul. Eng. 135, 73 (2009).

[28] E. Parteniades, Erosion and deposition of cohesive soil, J. Hydraul. Div. 91, 105 (1965).

[29] R. Ariathurai and K. Arulanandan, Erosion rates of cohesive soils, J. Hydraul. Div. 104, 279 (1978).

[30] J. S. Walder, Dimensionless erosion laws for cohesive sediment, J. Hydraul. Eng. 142, 04015047 (2015).

[31] L. A. Sanford and J. P.-Y. Maa, A unified erosion formulation for fine sediments, Mar. Geol. 179, 9 (2001).

[32] A. Knapen, J. Poesen, G. Govers, G. Gyssels, and J. Nachtergaele, Resistance of soils to concentrated flow eroison: A review, Earth-Sci. Rev. 80, 75 (2007).

[33] D. Marot, P.-L. Regazzoni, and T. Wahl, Energy-based method for providing soil surface erodibility rankings, J. Geotech. Geoenviron. 137, 1290 (2011).

[34] B. Indraratna, T. Muttuvel, and H. Khabbaz, Modelling the erosion rate of chemically stabilized soil incorporating tensile force-deformation characteristics, Can. Geotech. J. 46, 57 (2009).

[35] I. Haghighi, C. Chevalier, M. Duc, S. Guédon, and P. Reiffsteck, Improvement of hole erosion test and results on reference soils, J. Geotech. Geoenviron. 139, 330 (2012).

[36] J.-L. Briaud, F. C. K. Ting, H. C. Chen, Y. Cao, W. Han, and K. W. Kwak, Erosion function apparatus for scour rate predictions, J. Geotech. Geoenviron. 127, 105 (2001).

[37] N. Benahmed and S. Bonelli, Investigating concentrated leak erosion behaviour of cohesive soils by performing hole erosion tests, Eur. J. Environ. Civ. Eng. 16, 43 (2012).

[38] G. J. Hanson and K. R. Cook, Apparatus, test procedures, and analytical methods to measure soil erodibility in situ, Appl. Eng. Agric. 20, 455 (2004).

[39] P. Claudin and B. Andreotti, A scaling law for aeolian dunes on Mars, Venus, Earth and for subaqueous ripples, Earth Planet. Sci. Lett. 252, 30 (2006).

[40] C. Guidoux, Y.-H. Faure, R. Béguin, and H. Chia-Chun, Contact erosion at the interface between granular coarse soil and various base soils under tangential flow condition, J. Geotech. Geoenviron. 136, 741 (2010).

[41] W. Lick, L. Jin, and J. Gailani, Initiation of movement of quartz particles, J. Hydraul. Eng. 130, 755 (2004).

[42] F. Ternat, P. Boyer, F. Anselmet, and M. Amielh, Erosion threshold of saturated natural cohesive sediments: Modeling and experiments, Water Resour. Res. 44, W11434 (2008).

[43] S. T. Nase, W. L. Vargas, A. A. Abatan, and J. J. McCarthy, Discrete characterization tools for cohesive granular material, Powder Technol. 116, 214 (2001).

[44] A. Castellanos, The relationship between attractive interparticle forces and bulk behaviour in dry and uncharged fine powders, Adv. Phys. 54, 263 (2005).

[45] A. Anand, J. S. Curtis, C. R. Wassgren, B. C. Hancock, and W. R. Ketterhagen, Predicting discharge dynamics of wet cohesive particles from a rectangular hopper using the discrete element method (DEM), Chem. Eng. Sci. 64, 5268 (2009).

[46] A. Schmeink, L. Goehring, and A. Hemmerle, Fracture of a model cohesive granular material, Soft Matter 13, 1040 (2017).

[47] J.-Y. Delenne, V. Topin, and F. Radjaï, Failure of cemented granular materials under simple compression: Experiments and numerical simulations, Acta Mech. 205, 9 (2009). 
[48] F. Brunier-Coulin, P. Cuéllar, and P. Philippe, Erosion onset of a cohesionless granular medium by an immersed impinging round jet, Phys. Rev. Fluids 2, 034302 (2017).

[49] Z. Benseghier, P. Cuéllar, L.-H. Luu, J.-Y. Delenne, S. Bonelli, and P. Philippe, Relevance of free jet model for soil erosion by a laminar impinging jet in 2D, J. Hydraul. Eng. 146, 04019047 (2020).

[50] S. Beltaos and N. Rajaratnam, Impingement of axisymmetric developing jets, J. Hydraul. Res. 15, 311 (1977).

[51] D. Phares, G. T. Smeley, and R. C. Flagan, The wall shear stress produced by the normal impingement of a jet on a flat surface, J. Fluid Mech. 418, 351 (2000).

[52] S. M. Ghaneeizad, J. F. Atkinson, and S. J. Bennett, Effect of flow confinement on the hydrodynamics of circular impinging jets: Implications for erosion assessment, Environ. Fluid Mech. 15, 1 (2015).

[53] S. Badr, G. Gauthier, and P. Gondret, Erosion threshold of a liquid immersed granular bed by an impinging plane liquid jet, Phys. Fluids 26, 023302 (2014).

[54] H. Schlichting, Boundary Layer Theory (McGraw Hill Book Co., New York, 1960).

[55] F. Mercier, S. Bonelli, P. Pinettes, F. Golay, F. Anselmet, and P. Philippe, Comparison of computational fluid dynamic simulations with experimental jet erosion tests results, J. Hydraul. Eng. 140, 04014006 (2014).

[56] S. Kuang, C. LaMarche, J. S. Curtis, and A. Yu, Discrete particle simulation of jet-induced cratering of a granular bed, Powder Technol. 239, 319 (2013).

[57] P. Cuéllar, P. Philippe, S. Bonelli, N. Benahmed, F. Brunier-Coulin, J. Ngoma, J.-Y. Delenne, and F. Radjaï, Micromechanical analysis of the surface erosion of a cohesive soil by means of a coupled LBM-DEM model, in Proceedings of the 4th International Conference on Particle-Based Methods, PARTICLES2015, Barcelona, Spain, 28th-30th September 2008, edited by E. Oñate, M. Bischoff, D. R. J. Owen, P. Wriggers, and T. Zohdi (International Center for Numerical Methods in Engineering, Barcelona, Spain, 2008).

[58] P. Cuéllar, Z. Benseghier, L.-H. Luu, S. Bonelli, J.-Y. Delenne, and P. Philippe, Numerical insight into the micromechanics of jet erosion of a cohesive granular material, EPJ Web Conf. 140, 15017 (2017).

[59] A. Revuelta, A. L. Sánchez, and A. Liñán, The virtual origin as a first-order correction for the far-field description of laminar jets, Phys. Fluids 14, 1821 (2002).

[60] J. A. Dijksman, F. Rietz, K. A. Lörincz, M. van Hecke, and W. Losert, Refractive index matched scanning of dense granular materials, Rev. Sci. Instrum. 83, 011301 (2012)

[61] S. F. Wright, I. Zadrazil, and C. N. Markides, A review of solid-fluid selection options for optical-based measurements in single-phase liquid, two-phase liquid-liquid and multiphase solid-liquid flows, Exp. Fluids 58, 108 (2017).

[62] R. Béguin, P. Philippe, and Y.-H. Faure, Pore-scale flow measurements at the interface between a sandy layer and a model porous medium: Application to statistical modeling of contact erosion, J. Hydraul. Eng. 139, 1 (2013).

[63] P. Philippe and M. Badiane, Localized fluidization in a granular medium, Phys. Rev. E 87, 042206 (2013).

[64] See Supplemental Material at http://link.aps.org/supplemental/10.1103/PhysRevFluids.5.034308 for a summary of the entire experimental campaign carried out in the present study.

[65] N. Mitarai and F. Nori, Wet granular materials, Adv. Phys. 55, 1 (2006).

[66] B. Mielniczuk, O. Millet, G. Gagneux, and M. S. El Youssoufi, Characterisation of pendular capillary bridges derived from experimental data using inverse problem method, Granular Matter 20, 14 (2018).

[67] G. Lian, C. Thornton, and M. J. Adams, A theoretical study of the liquid bridge forces between two rigid spherical bodies, J. Colloid Interface Sci. 161, 138 (1993).

[68] N. P. Kruyt and O. Millet, An analytical theory for the capillary bridge force between spheres, J. Fluid Mech. 812, 129 (2017).

[69] O. Pitois, P. Moucheront, and X. Chateau, Rupture energy of a pendular liquid bridge, Eur. Phys. J. B 23, $79(2001)$

[70] J.-P. Gras, J.-Y. Delenne, and M. S. El Youssoufi, Study of capillary interaction between two grains: A new experimental device with suction control, Granular Matter 15, 49 (2013).

[71] G. I. Tardos and R. Gupta, Forces generated in solidifying liquid bridges between two small particles, Powder Technol. 87, 175 (1996). 
[72] J.-Y. Delenne, M. S. El Youssoufi, and F. Cherblanc, Mechanical behaviour and failure of cohesive granular materials, Int. J. Numer. Anal. Meth. Geomech. 28, 1577 (2004).

[73] R. Kirsch, U. Bröckel, L. Brendel, and J. Török, Measuring tensile, shear and torsional strength of solid bridges between particles in the millimeter regime, Granular Matter 13, 517 (2011).

[74] I. Cavarretta, I. Rocchi, and M. R. Coop, A new interparticle friction apparatus for granular materials, Can. Geotech. J. 48, 1829 (2011).

[75] K. Senetakis and M. R. Coop, The development of a new micro-mechanical inter-particle loading apparatus, Geotech. Test. J. 37, 1028 (2014).

[76] T.-H. Kim and C. Hwang, Modeling of tensile strength on moist granular earth material at low water content, Eng. Geol. 69, 233 (2003).

[77] V. Richefeu, L. S. El Youssoufi, and F. Radjaï, Shear strength properties of wet granular materials, Phys. Rev. E 73, 051304 (2006).

[78] V. V. Lemanov, V. I. Terekhov, K. A. Sharov, and A. A. Shumeiko, An experimental study of submerged jets at low Reynolds numbers, Tech. Phys. Lett. 39, 421 (2013).

[79] P. Pierrat and H. Caram, Tensile strength of wet granular materials, Powder Technol. 91, 83 (1997).

[80] C. Song, P. Wang, and H. A. Makse, A phase diagram for jammed matter, Nature (London) 453, 629 (2008).

[81] T. Aste, M. Saadatfar, and T. J. Senden, Geometrical structure of disordered sphere packings, Phys. Rev. E 71, 061302 (2005). 